\title{
ART ET RACE. LES GRAVURES DES PEUPLES PRIMITIFS À L'EXPOSITION UNIVERSELLE DE PARIS DE $1878^{*}$
}

\section{ART AND RACE. THE ENGRAVINGS OF PRIMI- TIVE PEOPLE AT THE 1878 PARIS WORLD'S FAIR}

\begin{abstract}
Based on a case study, this paper aims to examine the scientific, industrial and political interests that intertwine at the 1878 Paris World's Fair. We will focus on a graphic composition that was elaborated from various copies of rock art presented in several pavilions of the Exhibition and published by a science magazine. This figure was composed to compare the artistic capacities of European prehistoric and African contemporary primitives, all belonging, in the discourse of the French anthropologists, to the same race. The article considers the construction of anthropology in public space as a science claiming to be capable of analysing racial relationships in their environment and therefore capable of scientifically directing the French colonial project.
\end{abstract}

Keywords: Arthur Bordier, 1878 Paris World's Fair, rock art, race, colonialism, popular sciences, anthropology, prehistory, science museums.

\section{Introduction}

Malgré leur origine commerciale, les Expositions universelles ont été défi-

- Adresse pour correspondance: Centre Alexandre-Koyré, UMR 8560, EHESS/CNRS/MNHN, 27 rue Damesme, 75013 Paris, France. Email: maddalena.cataldi@ehess.fr.

*L'auteur tient à remercier les nombreux spécialistes qui ont participé, à différents titres, à ce travail. Les participants du congrès international Visibility matters à l'Université de Lucerne en 2013, spécialement Marianne Sommer, qui ont bien voulu discuter une première version. José Lanzarote Guiral et Anne Loyau qui ont organisé les journées d'études Montrer et démontrer la préhistoire à Paris en septembre 2013, où ce travail a été présenté Leur apport avisé a été fondamental pour la rédaction de ce texte, ainsi que leur généreux accord pour sa publication dans Organon. Claude Blanckaert et Arnaud Hurel avec lesquels j'ai pu discuter à diverses reprises, pour leur soutien indéfectible et précieux. Enfin, Michaël Gasperoni pour ses remarques stimulantes. 
nies comme des fêtes des classes populaires au décor conçu pour consacrer le progrès scientifique, technique et industriel. À partir de la Great Exhibition de 1851 à Londres, à travers la collaboration des administrations nationales et du secteur industriel, les Expositions universelles devinrent les lieux éphémères de la glorification du progrès technique européen et des produits de l'industrialisation ${ }^{1}$. En ce qui concerne plus particulièrement les sciences, ces expositions ont été l'occasion, par le biais de l'organisation simultanée de congrès scientifiques, de la mise en scène de leurs objets d'étude et de la célébration de leurs avancées, de consolider les disciplines ${ }^{2}$. C'est notamment le cas de l'anthropologie à l'Exposition universelle de Paris de 1878 .

En 1878, elle bénéficie, avec l'Exposition universelle, d'une vitrine pour consolider ses positions. Les anthropologues français participent à cette manifestation pour diffuser les connaissances de leur champ disciplinaire auprès du grand public et obtenir un soutien pour leurs projets à venir. Dans cette perspective, ils s'efforcent de souligner le potentiel des colonies et participent à l'élaboration d'instruments les plus à mêmes d'aider à l'exploitation des nouveaux territoires. Ils se mettent au service d'un projet national et patriotique. Ils contribuent ainsi à l'esprit de célébration républicaine qui anime l'Exposition, dont témoigne entre autres, en musique, la Chanson nouvelle patriotique L'ouverture de l'exposition universelle de Paris des ouvriers de Seclin (Lille):

Les délégués de chaque partie du monde

Viendront chez nous admirer nos exploits [...]

Débarqueront des Empereurs et des Rois

Oh! Liberté par ta sublime aisance

Donne aux monarques une belle leçon

Fraternité fait l'honneur de la France

À l'ouverture de notre Exposition [...]

Vous pourrez voir dans cette arène,

Tous les progrès de la civilisation

et vous verrez la France républicaine

À l'ouverture de notre Exposition. ${ }^{3}$

À partir d'un cas d'étude circonscrit mais représentatif des enjeux à l'œuvre, cet article entend mettre en lumière l'articulation entre les intérêts scientifiques, industriels et politiques qui colorent cet évènement. Nous allons concentrer notre attention sur un article et la figure qui l'accompagne, et qui regroupe plusieurs représentations rupestres présumées préhistoriques et des œuvres de peuples africains contemporains. Cette composition graphique fut élaborée par Arthur Bordier (1841-1910), anthropologue matérialiste de la Société d'Anthropologie de Paris (SAP), à partir de relevés présentés dans différents pavillons de l'Exposition Universelle et publiée dans La Nature, une revue de vulgarisation, cette même année 1878 .

\footnotetext{
${ }^{1}$ Cf. T. Bennett, The Birth of the Museum, p. 81.

${ }^{2}$ Cf. Ch. Demeulenaere-Douyère (éd.), Exotiques expositions ... \& A.-L. Carré \& M.-S. Corcy (éd.), Les expositions universelles en France au XIX $X^{e}$ siècle.

${ }^{3}$ L'ouverture de l'exposition universelle de Paris.
} 
L'analyse de cette image et du texte qu'elle illustre permet d'ouvrir une réflexion sur le rôle de cette Exposition dans les débats scientifiques qui ont traversé l'anthropologie française à propos des capacités cognitives des peuples préhistoriques et de ceux qui étaient appelés primitifs contemporains. Les relations mutuelles entre une race, sa capacité artistique et son potentiel évolutif définissent les contours de la discussion. Pour ce qui concerne plus spécifiquement l'art préhistorique, ce débat va occuper les anthropologues et les préhistoriens pendant le dernier quart du XIX ${ }^{\mathrm{e}}$ siècle. Notre analyse présente les positions des anthropologues matérialistes. Le militantisme revendiqué de ce courant de l'anthropologie française nous permet de considérer l'image que nous analysons comme un acte d'engagement social ${ }^{1}$. Ainsi, en démêlant les rapports entre exposition, vulgarisation et débats théoriques, il est possible de comprendre la construction de l'anthropologie matérialiste dans l'espace public à cette époque comme une science se revendiquant comme utile, capable d'analyser l'évolution d'une race dans son milieu et donc de diriger scientifiquement le projet colonial.

La construction de l'espace public de ce savoir fonctionne comme une comptabilité en partie double: l'équilibre de sa balance comptable se construit dans les apports mutuels des enjeux politiques et des enjeux scientifiques. Si le dernier fait appel aux scientifiques pour certaines prises de décision, les scientifiques ne sont pas imperméables aux contingences politiques de leur temps. De plus, notre étude de la construction d'un espace public pour la discipline se construit dans un paysage façonné par l'industrie de la presse et par ses acteurs, les scientifiques et les professionnels de la vulgarisation. Les croisements entre science et presse ont contribué, dès les années 1830, à nourrir la vision populaire de l'homme fossile ${ }^{2}$. Ces circonstances révèlent tout l'intérêt d'une analyse centrée sur l'illustration publiée dans La Nature.

La vulgarisation scientifique est, selon Bernadette Bensaude-Vincent, particulièrement tributaire du développement des nouvelles technologies de reproduction de l'illustration dans l'industrie de la presse ${ }^{3}$. Les historiens ont mis en lumière la centralité du rôle des illustrations scientifiques à partir des recherches fondatrices de Martin Rudwick ${ }^{4}$. Pour l'archéologie et la préhistoire plus spécifiquement, Stéphanie Moser et Nathan Schlanger ont étudié la généalogie des images scientifiques comme vecteurs de la construction d'une communauté épistémique apte à lire les informations incarnées dans ces illustrations ${ }^{5}$. A un moment où les images scientifiques commençaient à être largement relayées en dehors des revues disciplinaires, cette diffusion eut des

\footnotetext{
${ }^{1}$ Cf. C. Blanckaert, De la race à l'évolution, p. 24.

${ }^{2}$ L'histoire de l'homme fossile in: C. Blanckaert, Avant Adam. Cf. aussi O. Hochadel, M. Carandell Baruzzi \& C. Florensa, Scuffles, Scoops and Scams, un numéro spécial de Centaurus sur les relations entre presse généraliste et discipline préhistorique depuis les années 1870. Cf. aussi N. Coye, En leur science et conscience pour une discussion des rapports entre vulgarisation et préhistoire.

${ }^{3}$ Cf. B. Bensaude-Vincent, Un public pour la science ..., p. 55.

${ }^{4}$ Cf. M. J. S. Rudwick, Scenes from Deep Time.

${ }^{5}$ S. Moser, Making expert Knowledge through the Image \& N. Schlanger, Series in progress ... .
} 
répercussions sur l'image publique des sciences, sur l'attitude du public envers les sciences, et, finalement, une influence sur les scientifiques eux-mêmes dans leurs débats disciplinaires. Stéphanie Moser et Marianne Sommer ont pour leur part souligné l'importance de l'illustration populaire des hommes de Néanderthal dans la construction des idées scientifiques sur cette espèce humaine ${ }^{1}$. Les illustrations scientifiques ont été centrales dans la diffusion des connaissances et pour leur fonction d'intermédiaire, souvent créatif, entre la communauté scientifique et le public des sciences ${ }^{2}$. D'où l'intérêt de concentrer notre analyse sur cette représentation, laquelle fut conçue comme un élément à l'appui d'une théorie scientifique en débat.

Cette image compare en effet trois manifestations de l'art dolichocéphale, pour reprendre l'un des référents usuels de l'anthropologie du $\mathrm{XIX}^{\mathrm{e}}$ siècle. L'appartenance à cette race est le prérequis à la base de la comparaison, qui est à l'époque une véritable méthode de construction de la preuve scientifique. Dans le discours de l'anthropologie matérialiste, cette démonstration fondait l'appréciation du potentiel évolutif d'une race sur une évaluation de sa production esthétique.

L'illustration que nous analysons se construit à l'intersection de dynamiques historiquement situées: entre l'exposition d'objets (qui deviendront par la suite des objets muséaux), la publication d'articles dans la presse de vulgarisation et l'engagement social d'une science. Elle peut donc être comprise comme la matérialisation des relations entre les trajectoires plus générales des sociétés européennes: le mouvement de muséification des objets ethnographiques, la création d'une culture scientifique de masse et ses implications dans la politique coloniale française.

\section{L'Exposition universelle comme espace de travail pour la SAP \\ 2.1. L'Exposition internationale des sciences anthropologiques}

La Société d'Anthropologie de Paris fut chargée pour l'Exposition universelle de 1878 de l'organisation d'une section spécifiquement dédiée aux sciences anthropologiques ${ }^{3}$. La manifestation de 1878 s'inspira de celle de 1867 , où la préhistoire avait été exposée pour la première fois à un public général ${ }^{4}$. À cette date, agençant le musée rétrospectif de l'Histoire du Travail, le comité organisateur, dont Édouard Piette (1827-1906) et Gabriel de Mortillet (1821-1898) faisaient partie, avait abandonné le classement des objets par collectionneur, privilégié auparavant, pour une perspective comparatiste des produits du travail de l'homme aux diverses époques et chez les différents peuples $^{5}$. Les objets de la Galerie de l'Histoire du Travail étaient disposés dans

\footnotetext{
${ }^{1}$ S. Moser, The Visual Language of Archeology ... \& M. Sommer, Mirror, Mirror on the Wall ... .

${ }^{2}$ V. Chansigaud, Scientific Illustrators.

${ }^{3}$ A. Bordier, Notice sur l'exposition des sciences anthropologiques, p. 572.

${ }^{4}$ N. Müller-Scheessel, Fair Prehistory ..., pp. 391-394 \& Ch. Quiblier, L'exposition préhistorique de la Galerie de l'Histoire du travail en 1867.

${ }^{5}$ Cité après: Ch. Quiblier L'exposition préhistorique, p. 68. Au sujet de l'Exposition de 1867 voir les indications in: N. Schlanger, Boucher de Perthes au travail, pp. 281-282.
} 
l'ordre chronologique depuis la Gaule avant les métaux jusqu'à 1800, afin de mettre en avant le programme universaliste de l'exposition: l'histoire du travail, c'est l'histoire complète de l'humanité ${ }^{1}$. Les objets préhistoriques illustraient la première étape de la progression de la préhistoire aux machines compliquées qu'invente chaque jour le génie industriel du XIX $X^{e}$ siècle $^{2}$. En 1867, la préhistoire, démontrant la trajectoire coïncidente du développement humain et du progrès industriel, se trouvait également exposée au cour de l'espace de l'Exposition. Les préhistoriens ne disposaient pas d'un espace propre pour leur discipline, mais ils étaient chargés d'organiser leur section comme l'une des étapes, celle du point de départ, du parcours technique de l'humanité. En revanche, en 1878, la SAP acquiert une plus grande autonomie. Elle est chargée de l'organisation d'une section spécialement dédiée aux sciences anthropologiques et préhistoriques ${ }^{3}$.

Cette Société, fondée par Paul Broca (1824-1880) en 1859, avait fait l'objet d'un contrôle politique étroit sous Napoléon III. Il craignait alors son potentiel anticlérical et anti-autoritaire au point que ses réunions furent, semble-t-il, surveillées par la police ${ }^{4}$. Par la suite, elle trouva dans la III $^{\mathrm{e}}$ République naissante un terrain propice à son développement et un soutien plus large ${ }^{5}$. La Commission générale de l'Exposition de 1878 considérait la section de la SAP comme une æuvre d'initiative privée dont elle s'engageait à respecter l'autonomie ${ }^{6}$. Pour bien montrer que l'État $\mathrm{n}$ 'entendait prendre aucune responsabilité à l'égard des doctrines émises, la SAP fut autorisée à nommer elle-même les commissaires chargés de recevoir et de classer les objets présentés ${ }^{7}$. Déterminée à rendre service à la science plutôt qu'à fournir un spectacle au public, la SAP s'investit énergiquement dans la préparation de son espace d'exposition ${ }^{8}$. Finalement les matériaux rassemblés furent si nombreux que l'espace initialement prévu au cœur du Palais des Expositions du Champ de Mars s'avéra insuffisant. Le siège de l'Exposition internationale des sciences anthropologiques ${ }^{9}$ fut déplacé au Quai Billy (aujourd'hui avenue de New York), à la périphérie du reste de l'Exposition. La présentation de la discipline anthropologique (et préhistorique) de 1878, éphémère mais imposante, fut

\footnotetext{
${ }^{1}$ Cité après: Ch. Quiblier L'exposition préhistorique, p. 69.

${ }^{2}$ Cité après: Ch. Quiblier L'exposition préhistorique, p. 71 et p. 69.

${ }^{3}$ A. Bordier, Notice sur l'exposition des sciences anthropologiques, p. 572.

${ }^{4} \mathrm{H}$. Thulié, L'École d'anthropologie depuis sa fondation, p. 3.

${ }^{5}$ Cf. M. Hammond, Anthropology as a weapon of social combat in late-nineteenth-century France, p. 118.

${ }^{6}$ Exposition des sciences anthropologiques, 1881, p. 195.

${ }^{7}$ Exposition des sciences anthropologiques, 1881, p. 194

${ }^{8}$ A. Bordier, Notice sur l'exposition des sciences anthropologiques, p. 572

${ }^{9}$ Nom figurant sur les documents officiels de l'Exposition universelle. Cf. A. Bordier, Notice sur l'exposition des sciences anthropologiques, p. 572, mais on trouve plusieurs noms pour cette manifestation dont Galerie des sciences anthropologiques, Exposition de la Société d'anthropologie de Paris, Musée anthropologique, Musée d'anthropologie.
} 
considérée comme un triomphe par les organisateurs, même si certains rapports de l'époque invitent à nuancer ces propos ${ }^{1}$.

En 1878, l'éloignement de la SAP aux marges de l'Exposition traduit, nous l'avons vu, des circonstances ponctuelles. Toutefois, les espaces organisés par la $\mathrm{SAP}$ répondaient à une conception à la fois politique et scientifique de son fondateur, Paul Broca, qui n'était que partiellement partagée au sein de l'association. Comme pour d'autres disciplines naturalistes, la centralisation des données par le biais de la formation de collections (objets, représentations visuelles, données de mensurations, etc.) était essentielle à la construction du savoir anthropologique ${ }^{2}$. Mais, comme le précise Broca dans son allocution au congrès des sciences anthropologiques d'août 1878, les collections qui avaient été rassemblées dans les différents pays, étaient, à elles seules, incomplètes. Elles étaient souvent représentatives d'un seul sujet, à une seule époque, à une seule population. En outre, elles restaient généralement inaccessibles à la plupart des savants en raison de leur dispersion. Situation dommageable alors que, justement, la comparaison des spécimens et des collections était la base du travail des anthropologues ${ }^{3}$. Une Exposition universelle les rassemblant en grand nombre représentait donc une excellente occasion pour le travail de ces scientifiques. Elle ne fut pas manquée par la SAP. Mettant à profit la réunion de collections dispersées, elle se proposait d'en régulariser les procédés de description, de mensuration ou de représentation, de manière à rendre comparables entre eux les faits constatés par les divers observateurs ${ }^{4}$. Les sections de l'exposition de Paris étaient très largement composées de prêts de collectionneurs privés ${ }^{5}$. Plusieurs commentateurs se plaignirent d'ailleurs que les objets les plus raffinés prêtés par les collectionneurs aient abouti à la section consacrée à l'ethnographie des peuples étrangers à l'Europe et à l'art ancien de tous les pays, hébergée au Palais de Trocadéro, et les moins intéressants sur le plan esthétique à l'exposition des anthropologues de la SAP, au Quai Billy ${ }^{6}$. La SAP ne jugea pas utile d'exposer les collections des autres institutions parisiennes (laboratoire d'anthropologie du Muséum et Musée des antiquités nationales de Saint-Germain-en-Laye), pertinentes mais qui ne nécessitaient pas le travail normatif qu'elle se proposait d'effectuer; elles furent donc visitées par les participants au Congrès d'anthropologie au cours du mois août ${ }^{7}$. En définitive, on peut considérer que la SAP, sans pour autant représenter à elle seule la

\footnotetext{
${ }^{1}$ Cf. N. Müller-Scheessel, Fair Prehistory ..., p. 395. Cf. B. Noël, Les antiquités à l'exposition universelle de Paris, p. 83, pour des avis moins enthousiastes que celui des organisateurs.

${ }^{2}$ Pour la botanique cf. par ex. D. Bleichmar, Visible Empire ... ; sur le rôle et la construction des collections dans l'anthropologie française N. Dias, Nineteenth-Century French Collections of Skulls and the Cult of Bones.

${ }^{3}$ Cf. Séance d'ouverture ..., p. 20.

${ }^{4}$ A. Bordier, Notice sur l'exposition des sciences anthropologiques, p. 572.

${ }^{5}$ Cf. M. Charpy, Les «techniques archaïques», p. 284.

${ }^{6}$ B. Noël, Les antiquités à l'exposition universelle de Paris, p. 88. Au Trocadéro étaient exposées les antiquités européennes et l'art des sociétés exotiques.

${ }^{7}$ Cf. Séance d'ouverture ..., p. 24.
} 
discipline, marqua profondément cette exposition de son empreinte ${ }^{1}$. Bien qu'Armand de Quatrefages (1810-1892), titulaire de la chaire d'anthropologie du Muséum d'Histoire naturelle de Paris, fut aussi impliqué dans la Commission d'organisation de l'exposition anthropologique, celle-ci était en réalité entièrement prise en main par les membres de la SAP.

L'exposition de la SAP semblerait traduire l'attitude politique que Paul Broca envisageait pour l'anthropologie. Le fondateur de la SAP incitait en effet les anthropologues à garder leurs distances à l'égard des applications politiques, et aussi à se protéger des convoitises que le personnel politique pouvait avoir envers la discipline, pour se consacrer à la science pure ${ }^{2}$. Mais si Broca avait, pour le futur de la science anthropologique comme pour cette Exposition, des projets qui explicitaient l'exclusion d'une application de la discipline au domaine politique, d'autres anthropologues, et notamment les matérialistes affiliés à la SAP - tel Arthur Bordier, l'auteur du guide publié dans La Nature - revendiquaient une dimension résolument militante pour leur discipline. L'illustration que nous analysons est donc un indice de la multiplicité des activités du courant matérialiste de l'anthropologie française qui, tout en adhérant au programme de recherche de la SAP, menait en parallèle son propre agenda politico-scientifique.

Portée par sa volonté d'homogénéisation des mensurations, la SAP exposait, dans la section intitulée anthropologie générale et biologie, les instruments de mesure des anthropologues ${ }^{3}$. Dans cet espace l'on pouvait aussi voir les échantillons et les squelettes d'hommes adultes de différentes races, les crânes préhistoriques et actuels, les préparations d'organes et les moulages résumant, dans toute la classe des mammifères, l'histoire des circonvolutions cérébrales ${ }^{4}$. La section dédiée à l'anatomie anthropologique, qui fut définie comme l'ossuaire des Catacombes, présentait également une collection de grands singes empaillés ${ }^{5}$. Dans cette première section, une sous-partie sur la contribution des arts à l'anthropologie, organisée par Eugène Viollet-le-Duc (1814-1879), fut aussi proposée 6 . Une autre section, anthropologie préhistorique, exposait, en vue de retracer l'histoire physique de l'homme depuis son origine, les vestiges

\footnotetext{
I Pour l'histoire de la SAP en cette période cf. J.-C. Wartelle, La Société d'Anthropologie de Paris de 1859 à 1920 .

${ }^{2}$ Cependant, selon C. Blanckaert, La crise de l'anthropométrie ..., p. 152, les seules applications politiques tangibles de l'anthropologie en France furent finalement encouragées par Broca. Il s'agit de la réforme de la conscription, de l'acclimatation des races et de la typologie des criminels.

${ }^{3}$ Sur l'importance des collections anatomiques dans l'institutionnalisation de la discipline cf. N. Dias, Séries de crânes et armée de squelettes... .

${ }^{4}$ A. Bordier, Notice sur l'exposition des sciences anthropologiques, pp. 574-575.

${ }^{5}$ Collection des Guides Conty, L'Exposition en poche. Guide pratique, p. 152.

${ }^{6}$ A. Bordier, Notice sur l'exposition des sciences anthropologiques, p. 577. Sa réalisation reste à éclaircir. Le Guide Conty ne la mentionne pas et il ne nous a pas été possible de retrouver d'autres documents relatifs à celle-ci hors la référence de Bordier. Sur la conception naturaliste de l'histoire de l'architecture développée par Viollet-le-Duc cf. I. Flour, Les Moulages du Musée de sculpture comparée. Sur l'implication de Viollet-le-Duc dans la fondation du Musée d'Ethnographie de Trocadéro cf. N. Dias, Le Musée d'Ethnographie du Trocadéro.
} 
et les industries primitives de nos pères, les premiers rudiments de leur art ${ }^{1}$. C'est donc dans cette section qu'on pouvait admirer les objets des sites paléolithiques majeurs français, tels que Saint-Acheul, la Madeleine, Solutré et Laugerie.

Sur les murs de la Galerie des sciences anthropologiques furent affichées les copies de gravures rupestres préhistoriques provenant d'Algérie. Ces documents furent présentés par le docteur Gustave Bleicher (1838-1901), géologue alsacien et médecin de l'armée française en Algérie, où il avait visité les sites archéologiques du sud-Oranais, près de Thyout, en 1875 (cf. Figure 2$)^{2}$. Il avait communiqué ses découvertes à la Société des Sciences de Nancy en $1877^{3}$. Ces gravures représentent des scènes de chasse; des hommes armés d'arc et de flèches attaquent de grands bovidés. Elles furent reproduites par Bordier dans l'illustration de La Nature qui nous intéresse.

\subsection{D'autres espaces d'exposition pour la préhistoire: les espaces exotiques}

Les savants qui se consacraient spécifiquement à l'étude des cultures préhistoriques avaient une raison particulière de s'intéresser à l'Exposition; elle résidait dans le fait que la discipline préhistorique alors en voie de définition avait pourtant mis au point une méthode de recherche efficace fondée sur la comparaison entre cultures primitives disparues et cultures sauvages actuelles. Dans la perspective du comparatisme ethnographique, l'étude des mœurs des sauvages actuels devenait un moyen pour appréhender les coutumes préhistoriques, car elle permettait d'éclairer les résultats de l'archéologie et de structurer le discours scientifique sur la société de l'homme fossile ${ }^{4}$. Ici aussi, l'Exposition universelle pouvait parfaitement servir ce but puisque se trouvaient réunis en un même lieu des objets de différentes provenances et chronologies. En effet, la SAP avait aussi organisé dans son bâtiment une section consacrée à l'ethnologie, ethnographie et linguistique, où elle exposait les échantillons d'industries locales, des photographies des différents types de races, des cartes de diffusion des langues et des dialectes ${ }^{5}$. Le but de cette section était de tracer une histoire des migrations construite sur les analogies des mours et affinités de race, à travers des objets qui indiquaient, chez les différents peuples, le degré et la nature de la civilisation ${ }^{6}$.

En France, l'ethnologie était considérée comme une discipline descriptive comprise dans l'anthropologie. Pour Broca, par exemple, elle était consacrée à l'étude des groupes partiels de l'humanité, alors que l'anthropologie générale étudiait le genre humain dans son ensemble et dans ses relations avec les autres

\footnotetext{
${ }^{1}$ A. Bordier, Notice sur l'exposition des sciences anthropologiques, p. 576.

2 Toutes les figures ont été placées à la fin de l'article.

${ }^{3}$ Cf. G. Bleicher, Extrait des procès-verbaux de la Société des sciences de Nancy ..., , p. 10.

${ }^{4}$ Cf. A. Hurel, Des «Bushmen» en Europe?, pp. 297-310.

${ }^{5}$ A. Bordier, Notice sur l'exposition des sciences anthropologiques, p. 575.

${ }^{6}$ A. Bordier, Notice sur l'exposition des sciences anthropologiques, p 575. Au total, les objets se divisaient en cinq sections. Suivaient la quatrième, démographie, et une dernière section dédiée à la bibliographie. De plus, une partie de l'espace était réservée à des expositions nationales, organisées par des sociétés savantes étrangères. Cf. A. Bordier, Les sciences anthropologiques à l'exposition universelle, pp. 408-410.
} 
animaux (anthropologie zoologique). Nous avons vu que cela se traduisait par l'exposition d'objets de la section anthropologie générale et biologie.

Les informations anatomiques et historiques sur ces groupes partiels pouvaient être recueillies par les voyageurs et faire l'objet par la suite des analyses de spécialistes de l'anthropologie, de la linguistique et de l'histoire, etc. ${ }^{1}$ En effet, jusqu'aux années 1880 , bien que l'intérêt du public ne cessât de croître, les objets ethnographiques n'avaient pas encore réuni une communauté de praticiens spécialement consacrés à leur étude ${ }^{2}$. À défaut de ces spécialistes, ce furent, selon Nélia Dias, les Expositions universelles qui ouvrirent la voie aux installations des musées d'ethnographie contemporains dans les villes européennes ${ }^{3}$.

Ce fut justement le cas à Paris. Des objets exotiques étaient exposés dans la Salle des Missions scientifiques - missions financées par le ministère de l'Instruction publique -, située au premier étage du Palais de l'Industrie du Champ de Mars. Cet espace, également dénommé Musée ethnographique, accueillait les objets et moulages collectés aux frais de ce ministère partout dans le monde. Cette collection constituera le premier noyau du Musée d'ethnographie fondé en 1879, ouvert en 1882 à Trocadéro et dirigé par le successeur de Quatrefages au Muséum, Ernest-Théodore Hamy (1842-1908) ${ }^{4}$.

Dans le futur musée, comme dans cette salle, l'ethnographie était, aux yeux des spécialistes, inséparable de l'archéologie préhistorique parce que les deux visaient à éclaircir la propagation des premières populations sur la terre, et [...] la première colonisation de l'Europe ${ }^{5}$. Les musées d'ethnographie permettaient d'établir des comparaisons illimitées entre les degrés de civilisation primitive des populations existantes ou éteintes du monde entier ${ }^{6}$.

Il n'est donc pas étonnant de trouver des objets préhistoriques dans les espaces consacrés à l'ethnographie. Sur les escaliers qui conduisaient à l'entrée principale de cette Salle des missions scientifiques, Émile Rivière (1835-1922) exposait les moulages de gravures sur rochers des Lacs des Merveilles (fig. 4) ${ }^{7}$, une carte de la zone et une vue panoramique de la vallée dessinée par son

${ }^{1}$ Cf. F. Zerilli, Il lato oscuro dell'etnologia ..., pp. 7-8.

${ }^{2}$ Cf. E. Sibeud, La fin du voyage.

${ }^{3}$ Cf. N. Dias, Le Musée d'Ethnographie du Trocadéro, p. 95.

${ }^{4}$ Cf. E.-Th. Hamy, Les origines du Musée d'ethnographie, pp. 56 et passim, affirme que cette Salle des Missions avait été conçue par le directeur de la division Sciences et Lettres du ministère, Oscar de Watteville (1824-1901), comme une preuve générale pour tester l'intérêt du public envers ce type d'objets et donc l'intérêt à en faire un Musée exotique permanent dans la capitale. Cf. aussi les discours de l'inauguration présentés dans Notice sur le Muséum d'ethnographie des missions scientifiques ..., pp. 3-8. Sur la fondation du Musée d'ethnographie du Trocadéro et le rôle d'Hamy comme organisateur des collections mésoaméricaines voir la plus récente des œuvres sur cette institution, le premier chapitre de A. Conklin, In the Museum of Man \& N. Dias, Le Musée d'Ethnographie du Trocadéro.

5 L'archéologue danois Jens Jacob Asmussen Worsaae (1821-1885) cité in: J. M. B. de Watteville, Circulaire relative à la création d'un musée ethnographique, pp. 897-898.

${ }^{6}$ Jens Jacob Asmussen Worsaae cité dans J. M. B. de Watteville, Circulaire relative à la création d'un musée ethnographique, p. 898.

${ }^{7}$ Les lacs des Merveilles se situent dans la Vallée des Merveilles, dans les Alpes du sud dans le massif du Mercantour à la frontière entre la France et l'Italie. 
adjoint ${ }^{1}$. Les moules avaient été réalisés dans le cadre d'une mission à l'étranger (Italie) financée par le ministère de l'Instruction publique en $1877^{2}$. Nous savons que les moulages exposés représentaient des armes en silex, des javelots en bronze et aussi des signes indéchiffrables, des carrés, des ronds ${ }^{3}$. En outre, dans un autre petit stand de la première salle, étaient exposés des spécimens de la faune, des photographies des grottes de Menton et des squelettes humains précédemment mis au jour par Rivière ${ }^{4}$. Rivière exposait dans la Salle des Missions scientifiques les résultats de trois missions menées par lui en Italie.

Beaucoup d'autres pavillons exposaient des pièces d'intérêt pour la discipline; comme l'anthropologue Arthur Bordier (1841-1910) le souligne dans son Rapport sur l'Ethnologie de l'Asie orientale, de l'Afrique et de l'Océanie, lu au Congrès d'anthropologie organisé en août, les stands nationaux contenaient des collections arrivées des colonies pouvant intéresser les anthropologues et les préhistoriens ${ }^{5}$. Par exemple, au sein du bâtiment central de l'Exposition au Champ de Mars, de l'autre côté de la Seine par rapport à la Galerie des sciences anthropologiques, l'Angleterre présentait les produits coloniaux de la colonie du Cap de Bonne-Espérance. Les objets des peuples sauvages ne bénéficiant d'aucun espace propre au sein des expositions et, après l'Exposition universelle de Paris en 1855, ils étaient habituellement présentés en tant que compléments subordonnés des stands nationaux ${ }^{6}$. Selon l'historien de la muséographie Tony Bennett, cela correspond à la transcription, au point de vue muséographique, du rapport entre progrès racial et progrès technique implicite dans la culture contemporaine et suggéré par le langage muséographique propre à ces évènements, sur lesquels nous reviendrons ${ }^{7}$. Un chroniqueur décrit ainsi le stand de la colonie du Cap de Bonne-Espérance:

Ce n'est pas grand, bien moins grand que ne le comporteraient les richesses incroyables réunies dans ce petit coin du Palais. [...] en levant les yeux, on aperçoit des gerbes magnifiques de plumes d'autruche; en regardant plus près de terre, on voit tout autour de soi l'ivoire d'énormes dents d'éléphants, et au milieu de tout, une petite vitrine bien simple où s'étalent plusieurs centaines de diamants bruts, parmi lesquels un

\footnotetext{
${ }^{1}$ E.-Th. Hamy, Les origines du Musée d'ethnographie, p. 57 et Ministère de l'Instruction publique, Exposition Universelle 1878, p. 29.

2 1871/1872, 1872/1873 aux grottes de Menton et 1877 aux Lacs des Merveilles, AN, Ministère de l'Instruction publique, F 17, 3003, A. Cf. É. Rivière, Gravures sur rocher du Lac des Merveilles ... .

${ }^{3}$ L. Simonin, Les Missions scientifiques, p. 605.

${ }^{4} \mathrm{Cf}$. Notice sur le Muséum d'ethnographie des missions scientifiques, pp. 9-10 \& Ministère de 1'Instruction publique, Exposition Universelle 1878, p. 29.

${ }^{5}$ A. Bordier, Rapport sur l'Ethnologie de l'Asie orientale, de l'Afrique et de l'Océanie, pp. 39-47.

${ }^{6}$ M. Charpy, Les «techniques archaïques», p. 283.

${ }^{7}$ T. Bennett, The Birth of the Museum, pp. 80-81.
} 
certain nombre formeront, quand l'art y aura passé, des bijoux royaux. ${ }^{1}$

C'est justement sur les murs de cet espace qu'étaient présentées des copies des gravures rupestres des Boschimans (fig. 3), un des peuples indigènes de la région, reproduites par Edward John Dunn (1844-1937). Dunn, géologue du Geological Survey of the Mines Department anglais, avait travaillé à la réalisation de la carte géologique de la colonie, et participé ainsi à la découverte de ses mines d'or et de diamants. Collectionneur et anthropologue, il s'était intéressé aux Boschimans, auxquels il dédiera une monographie en $1931^{2}$.

Pour conclure sur l'exposition d'objets préhistoriques, on pourra noter que, par l'analyse de la mise en exposition de ces derniers dans les Expositions universelles, il est possible de voir s'incarner une multiplicité de discours, à la fois politiques et scientifiques, soutenus par la muséographie; il est notamment intéressant d'en remarquer la coexistence au sein de cette manifestation.

En effet, d'une part, encadrés dans le contexte de l'Exposition universelle, les objets préhistoriques comparés aux outils des sauvages actuels prouvaient la coïncidence entre accomplissement culturel universel et progrès européen. Selon Emmanuelle Sibeud, la leçon des choses administrée par les objets exotiques exposés, agissait comme un miroir, justifiant la conquête des territoires étrangers et alimentant en même temps, chez les visiteurs des villes européennes, la foi dans le progrès qui caractérisait l'idéologie républicaine ${ }^{3}$.

Mais d'autre part, il faut aussi souligner que le discours sur le progrès de la civilisation pouvait servir une perspective nationaliste, où l'antiquité humaine comptait pour antiquité de la nation et où les objets préhistoriques enracinaient l'actualité industrielle dans le passé national. C'est le cas par exemple des vitrines composées par le producteur de céramique anglais Wedgwood, qui, juxtaposant ses produits à des tessons de poterie préhistorique anglaise, démontrait l'émergence d'un génie national supposé de la fabrication des céramiques ${ }^{4}$.

\section{Gravures ante-historiques sur rochers}

\subsection{Circonstances de la publication}

En analysant la Figure 1, nous allons examiner en détail un exemple de la démarche comparatiste que nous avons évoqué. Cette composition est réalisée à partir de dix éléments réunis dans la même planche. Ils appartiennent à trois groupes: des gravures pariétales préhistoriques provenant d'Algérie, des gravures œuvres de Boschimans contemporains et des gravures préhistoriques du Lac des Merveilles, situé à la frontière entre l'Italie et la France. Les modèles ayant servi à cet assemblage sont des copies présentées dans les différents pavillons de l'Exposition que nous venons de présenter. Nulle part ailleurs que dans cette illustration une telle association visuelle n'est faite. Légendée

\footnotetext{
${ }^{1}$ De la Blanchère, Les Diamants du Cap, p. 729.

${ }^{2}$ Cf. E. J. Dunn, The Bushman.

${ }^{3}$ Cf. E. Sibeud, La fin du voyage, p. 179.

${ }^{4}$ M. Charpy, Les «techniques archaïques», p. 286.
} 
Gravures ante-historiques sur rochers. Spécimens des échantillons anthropologiques de l'Exposition universelle, elle illustre un article d'Arthur Bordier intitulé Les sciences anthropologiques à l'exposition universelle publié dans La Nature.

Bordier conçoit cet article comme un guide de visite et oriente les visiteurs parmi l'importante quantité de spécimens anthropologiques rassemblés par la SAP et les autres objets d'intérêt pour la discipline. En rédigeant son article, Arthur Bordier pouvait suivre l'exemple de l'un des plus éminents préhistoriens français, Gabriel de Mortillet (1821-1898) qui avait construit ses Promenades préhistoriques à l'exposition universelle de 1867 sur ce principe ${ }^{1}$ : se déplaçant entre les différents stands nationaux de l'Exposition, Mortillet présentait pièce en main, les objets les plus importants pour la compréhension de plusieurs idées et appréciations qui me sont particulières ${ }^{2}$. Ces idées trouvaient une synthèse dans les lois énoncées à la fin du guide: la loi - matérialiste - du progrès de l'humanité, celle du développement similaire et leur présupposé: la haute antiquité de l'Homme ${ }^{3}$. Ces lois postulaient que toute l'humanité, depuis son origine lointaine, passerait par les étapes de la pierre taillée à éclats, pierre polie, bronze, fer, de la civilisation technique primitive, jusqu'à arriver à notre civilisation $^{4}$. Les expositions universelles fournissaient aux anthropologues l'occasion de construire un discours sur le progrès dans le long terme, des origines de l'humanité jusqu'à la modernité. Ce discours était diffusé au travers de publications scientifiques mais aussi par le biais d'éditions de vulgarisation, et de façon si large que le Commissaire de l'Exposition, Jean-Baptiste Krantz (1817-1899), affirma que, pour le grand public, la science anthropologique était née à l'Exposition de $1878^{5}$.

Arthur Bordier, était un médecin originaire de la Sarthe, formé à Paris. Membre de la SAP et du groupe matérialiste constitué autour de Mortillet, il joua un rôle de premier plan dans l'organisation de l'exposition des sciences anthropologiques, où il fut notamment chargé des vitrines de l'ethnographie générale, pour lesquelles il obtint une médaille et un diplôme ${ }^{6}$. Sa Notice sur l'exposition des sciences anthropologiques, dans le Rapport administratif, témoigne de son investissement dans cet évènement. Il faisait d'ailleurs partie de la Commission d'organisation du Congrès d'anthropologie.

Son travail se développait dans un cadre large qui comprenait d'autres branches de l'anthropologie, telles que l'hygiénisme ou l'anthropologie criminelle. En 1877, il fut le fondateur de la Société de médecine publique et d'hygiène professionnelle et, en 1878, il était nommé titulaire de la nouvelle

${ }^{1}$ Cf. G. de Mortillet, Promenades préhistoriques à l'exposition universelle et aussi comme livre: G. de Mortillet, Promenades préhistoriques à l'exposition universelle.

${ }^{2}$ G. de Mortillet, Promenades préhistoriques à l'exposition universelle, p. 1. Cf. M. Sommer, Human tools of the European tertiary? analyse le cas de la classification des éolithes dans le cadre de l'Histoire du travail de l'Exposition Universelle de 1867, à Paris, par Gabriel de Mortillet.

${ }^{3}$ G. de Mortillet, Promenades préhistoriques à l'exposition universelle, p. 187

${ }^{4}$ G. de Mortillet, Promenades préhistoriques à l'exposition universelle, p. 185.

${ }^{5}$ N. Müller-Scheessel, Fair Prehistory ..., p. 395.

${ }^{6}$ A. Picaud, Le docteur Arthur Bordier, pp. 12-13. 
chaire de géographie médicale à l'École d'anthropologie de Paris, qu'il occupa jusqu'en 1895.

La publication dans laquelle il écrivait était une revue de vulgarisation, fondée en 1873 par Masson, l'une des plus importantes maisons d'édition scientifique. Ce bulletin périodique fait partie des publications participant à l'essor de la vulgarisation des sciences en France dans la seconde moitié du XIX ${ }^{\mathrm{e}}$ siècle, un processus lié au succès des Expositions universelles ${ }^{1}$. La Nature fut ainsi reconnue d'utilité publique et distribuée dans les bibliothèques municipales et scolaires dès $1874^{2}$.

Son directeur, Gaston Tissandier exposait, dans la préface du premier numéro, la ligne éditoriale de sa revue, dans laquelle l'illustration avait une place centrale $^{3}$. Il s'agissait d'une revue richement illustrée avec des gravures de qualité, qui participa ainsi à l'essor des arts graphiques de la seconde partie du $\mathrm{XIX}^{\mathrm{e}}$ siècle.

Journal hebdomadaire d'actualité scientifique, La Nature voulait s'affranchir des cloisonnements disciplinaires des revues spécialisées. Tissandier rassemblait ainsi les travaux de différents spécialistes, afin de fuir l'écueil de l'erreur et de l'inexactitude ${ }^{4}$. Il souhaitait éviter de froisser toute susceptibilité, et concevait la science comme le plus sûr terrain de la conciliation et non comme un champ de bataille, encore moins comme l'arme d'un parti politiques. Et en effet, pour ce qui concerne l'anthropologie et la préhistoire, y écrivirent les spécialistes majeurs de l'époque, toutes appartenances scientifiques et politiques confondues, tels que Ernest-Théodore Hamy, Armand de Quatrefages, Gabriel de Mortillet, Félix Garrigou (1835-1920), Jacques Bertillon (1851-1922), Julien Girard de Rialle (1851-1904), Bordier et bien d'autres. Tissandier exhortait à dépasser les rancunes et les préjugés des partis, pour ne songer qu'au travail et aux progrès qui en dérivent ${ }^{6}$. Son but était donc patriotique: trouver de nouveaux adeptes de la science sur le modèle des scientifiques et vulgarisateurs anglais tel que John Tyndall (1820-1893) ou Michel Faraday (1791-1867), ce qui pourrait dissiper les ténèbres qui avaient conduit la France à la défaite de $1870^{7}$. Pour les auteurs, cette revue représentait un espace où défendre, face au public, leurs positions, sans la médiation du

\footnotetext{
${ }^{1}$ Le verbe vulgariser est encore qualifié de néologisme en 1881 par le dictionnaire Littré. Cf. B. Béguet, $L a$ science pour tous ..., p. 7. Sur les Expositions Universelles et la vulgarisation des sciences cf. Bernadette Bensaude-Vincent, Un public pour la science ..., p. 57.

${ }^{2}$ Qualifiée de modèle prestigieux et de référence incontestable par l'historien B. Béguet, La Nature perdura jusqu'en 1964 sous son nom d'origine avant d'être absorbée par La Recherche en 1973. B. Béguet, La science pour tous ..., p. 92.

${ }^{3}$ G. Tissandier, Préface, p. VII.

${ }^{4}$ G. Tissandier, Préface, p. V \& VI.

${ }^{5}$ G. Tissandier, Préface, p. VII.

${ }^{6}$ G. Tissandier, Préface, p. VII.

${ }^{7}$ G. Tissandier, Préface, p. VII. L'emploi de la vulgarisation par Tyndall a été analysé in Th. Gieryn, Boundary-Work and the Demarcation of Science from Non-Science ... .
} 
journalisme scientifique alors émergent et qui suivait son propre agenda scientifique et politique ${ }^{1}$.

\subsection{La démonstration de Bordier: comparaisons de l'art dolichocéphale}

D'un point de vue conceptuel, Bordier basait sa comparaison sur l'appartenance des auteurs de ces gravures au même groupe racial, celui des dolichocéphales. C'était une pratique commune en anthropologie. ErnestThéodore Hamy, dans son Précis de paléontologie (1870), comparait les outils les plus anciens retrouvés en Europe et les outils des sauvages océaniens contemporains et des Maoris ${ }^{2}$.

Mais cette démonstration était aussi fondée sur une appréciation esthétique qui prenait avant tout en compte la précision dans le rendu des représentations. Les gravures algériennes de Thyout présentées par Bleicher à l'exposition de la SAP, étaient décrites par Bordier comme l'œuvre naïve d'un peuple de chasseurs, qui avait voulu graver ses propres traits et ceux de ses ennemis ${ }^{3}$.

Bordier décrivait ensuite les quatre œuvres des Boschimans représentant des scènes de chasse avec des êtres humains et des animaux. Dans son article, Bordier soulignait les éléments des gravures qui mettent en valeur les caractéristiques anatomiques de ce peuple, telles que la petitesse de la tête, la stéatopygie, une marche non complètement redressée: il s'agissait donc d'une imitation fidèle de la réalité (cf. Figure 3$)^{4}$. Ces caractères anatomiques étaient d'ailleurs bien connus des visiteurs du Muséum national d'Histoire naturelle où la Vénus hottentote (le moulage du corps de Saartjie Baartman) était exposée depuis 1816 et que Bordier invitait à découvrir ${ }^{5}$. Les gravures des Boschimans étaient considérées par Bordier comme la preuve des qualités artistiques de ce peuple. Néanmoins, dans le discours de Bordier, les Boschimans se distinguaient par leur dégradation, comme le démontrait le fait que leur civilisation primitive disparaissait chaque jour devant les civilisations anglaise et hollandaise qui les enserrent ${ }^{6}$.

Enfin, les gravures des Lacs des Merveilles étaient décrites par Bordier comme grossières et démontrant une intelligence beaucoup moindre de la nature par rapport à celles des Boschimans: Eh bien, celles-là [les gravures du Lac des Merveilles relevées par Rivière], précisément, sont l'œuvre d'une population beaucoup plus avancée, plus intelligente et qui, pour avoir disparu, $n$ 'a pas été sans son jour de splendeur (cf. Figure 4) ${ }^{7}$. Ce peuple, appartenant à

${ }^{1}$ L'article de C. Blanckaert, Les bases de la civilisation ..., analyse ce type de posture de Mortillet envers Louis Figuier. Sur l'émergence du groupe professionnel des journalistes scientifiques et les enjeux liés à la vulgarisation de l'anthropologie préhistorique cf. M. Cataldi, Inventing the Menton Man.

${ }^{2}$ E.-Th. Hamy, Précis de paléontologie humaine, pp. 243-245.

${ }^{3}$ A. Bordier, Les sciences anthropologiques à l'exposition universelle, p. 359.

${ }^{4} \mathrm{Cf}$. A. Bordier, Les sciences anthropologiques à l'exposition universelle, p. 335.

${ }^{5}$ Cf. A. Bordier, Les sciences anthropologiques à l'exposition universelle, pp. 359-360. Une analyse du comparatisme en préhistoire (entre les venus préhistoriques et la Vénus hottentote) se trouve in: A. Hurel, Des «Bushmen» en Europe?

${ }^{6}$ A. Bordier, Les sciences anthropologiques à l'exposition universelle, p. 359.

${ }^{7}$ A. Bordier, Les sciences anthropologiques à l'exposition universelle, p. 360. 
la race des Cro-Magnon aurait été, à une période de la préhistoire, le colonisateur de toute la zone méditerranéenne. Un témoignage ultérieur de son expansion serait offert par les vases et les momies Guanches retrouvés aux Îles Canaries, également exposés dans le pavillon des Missions scientifiques, grâce aux expéditions de René Verneau ${ }^{1}$.

Dans le discours de l'anthropologie française, les dolichocéphales de CroMagnon avaient été précédés, sur le territoire européen, par l'homme $d u$ Neanderthal. Considéré auparavant comme un individu pathologique à cause de la morphologie massive de son crâne, le Néandertalien était devenu, avec la multiplication des découvertes, le prototype de la première race dolichocéphale installée en Europe depuis la fin du tertiaire ${ }^{2}$. La présence de l'homme dans l'Europe tertiaire étant encore objet de débats, les hommes de Neanderthal figuraient comme les premiers habitants du continent. Les Cro-Magnon ${ }^{3}$ furent une autre race dolichocéphale, plus accomplie du point de vue de la civilisation et de l'anatomie, succédant en Europe aux Néandertaliens, à la période de transition entre l'Âge du Mammouth et du Renne ${ }^{4}$. La conceptualisation de la fin du quaternaire et le passage de l'industrie de la pierre à celle des métaux étaient sujets à discussions à la SAP, même si la succession d'une race dolichocéphale autochtone et d'une race brachycéphale, à cheval sur ces deux époques, était largement acceptée 5 .

Selon Bordier, une certaine évolution était possible au sein d'une race, comme le démontrait le cas des dolichocéphales, les Boschimans - très dolichocéphales - étant moins développés au point de vue technique que les habitants préhistoriques de la Vallée des Merveilles. Toutefois, face à une race dont la technique était supérieure, les premiers auraient succombé ${ }^{6}$. Dans le discours de Bordier, les Boschimans étaient plus doués sur le plan artistique et moins sur le plan technique. Les dolichocéphales du Lac des Merveilles étaient au contraire plus avancés du point de vue technique, mais plus grossiers dans leur forme expressive. Ce peuple, aux dires de Bordier, avait pourtant dû subir l'invasion des peuples de race brachycéphale, les vivaces agriculteurs à la tête carrée venus coloniser l'Europe ${ }^{7}$.

\section{La disparition des dolichocéphales et la colonisation scientifique}

Ainsi, bien que les dolichocéphales Cro-Magnon fussent plus avancés que

\footnotetext{
${ }^{1}$ Cf. E.-Th. Hamy, Les origines du Musée d'ethnographie, p. 60.

${ }^{2}$ E.-Th. Hamy, Précis de paléontologie humaine, p. 242.

${ }^{3}$ Par référence au type défini après la découverte des squelettes de cinq individus dans l'abri Cro-Magnon des Eyzies-de-Tayac en 1868.

${ }^{4}$ E.-Th. Hamy, Précis de paléontologie humaine, pp. 272-281. Hamy reprend l'étude de Broca qui est la référence pour Cro-Magnon à l'époque. Les époques du Mammouth et de la Renne correspondent au milieu et à la fin du quaternaire. Cette succession des époques conceptualisée par la succession de la faune proposé par Lartet sera substitué par une datation basée sur les cultures préhistoriques proposée par Mortillet.

${ }^{5}$ Voir le chapitre consacré à ce sujet in: C. Blanckaert, De la race à l'évolution.

${ }^{6} \mathrm{Cf}$. A. Bordier, Les sciences anthropologiques à l'exposition universelle, p. 559.

${ }^{7}$ A. Bordier, Les sciences anthropologiques à l'exposition universelle, p. 362.
} 
les Boschimans actuels, leur disparition était inévitable, selon Bordier, face aux développements techniques mis en œuvre par la race brachycéphale survenue au Néolithique. Parmi les théories sur l'ethnogenèse européenne courantes au sein de la SAP, l'arrivée des peuples néolithiques, identifiée ou non avec l'installation d'une race différente de celle des autochtones, faisait l'objet de controverse. Les vues que Bordier partageait avec son public n'étaient pas majoritaires au sein de la $\mathrm{SAP}$, où aucune ligne idéologique concernant le potentiel évolutif des races ne prévalait ${ }^{1}$. En revanche, selon Claude Blanckaert, la position de Bordier était largement admise chez les matérialistes ${ }^{2}$. Mortillet acceptait l'arrivée d'une minorité brachycéphale des plateaux indiens, qui aurait colonisé culturellement l'Europe primitive avec certaines de ses coutumes, telles que la maîtrise du bronze. Il était plus réticent s'agissant de certaines races sauvages actuelles, notamment des Arabes peuplant l'Algérie ${ }^{3}$. D'autres furent moins optimistes. Pour le linguiste matérialiste Charles Letourneau (18311902) le développement moral et intellectuel était impossible sans le développement corrélatif des centres nerveux dont il était l'expression ${ }^{4}$. Pour ces matérialistes de différentes sensibilités politiques, les civilisations détruisent ce qu'elles ne peuvent pas emporter dans leur progression et certaines races étaient vouées à disparaitre, ne pouvant pas s'adapter aux avancées de la civilisation des races européennes ${ }^{5}$. Bordier indiquait un autre des objets exposés dans le stand de la colonie du Cap, afin d'étayer son propos. Il s'agissait d'une

[...] pointe de sagaie emmanchée, que je qualifierai volontiers de type de Moustier; mais cette pointe est en verre à bouteille, et la sertissure est faite d'un vieux clou de fer aplati, le tout trouvé sans doute parmi les débris de la civilisation voisine. Nous voyons ainsi que le fer a beau s'offrir, que le verre a beau présenter, devant une population qui n'est encore mûre que pour l'âge de la pierre mal taillée: ni le verre, ni le fer ne prennent aux yeux de cette population leur valeur réelle; ils ne lui servent qu'à titre d'objet dur ou tranchants. Cette arme de fer n'est pas moins un spécimen de l'âge de la pierre taillée. ${ }^{6}$

\footnotetext{
${ }^{1}$ Cf. C. Blanckaert, La crise de l'anthropométrie, p. 143. Voir par exemple les conceptions dichotomiques de Broca et Joseph Arthur de Gobineau au sujet du potentiel évolutif des peuples in: C. Blanckaert, $L a$ «météorologie du microcosme cérébral», p. 279.

${ }^{2}$ Cf. C. Blanckaert, La crise de l'anthropométrie, p. 139. Par contre Bordier serait le premier à juger certains êtres humains des non-valeurs. Cf. Claude Blanckaert, La crise de l'anthropométrie, p. 143.

${ }^{3}$ C. Blanckaert, La crise de l'anthropométrie, p. 138. Paul Topinard était de la même opinion. Sur la construction de l'assimilabilité des Kabyles et des Berbères et l'impossibilité d'assimilation des Arabes à partir des années 1840 dans le regard de la médecine coloniale française G. Boetsch \& J.-N. Ferrie, Le paradigme berbère ....

${ }^{4}$ Cité après: C. Blanckaert, La crise de l'anthropométrie, p. 138

${ }^{5}$ Cf. C. Blanckaert, La crise de l'anthropométrie, p. 139.

${ }^{6}$ A. Bordier, Rapport sur l'Ethnologie de l'Asie orientale, de l'Afrique et de l'Océanie, p. 43.
} 
En effet, Bordier, en soulignant l'inévitable disparition des artistes dolichocéphales Boschimans face à la supériorité technique occidentale, visait à indiquer à ses collègues que les Boschimans ne pouvaient faire partie des races susceptibles de se mélanger aux Européens dans les colonies. En outre, Bordier indiquait aux lecteurs de La Nature le rôle que l'anthropologie pouvait assurer dans la France contemporaine, celui d'une science utile à la colonisation.

En effet, le Congrès d'Anthropologie, réuni à Paris durant l'été de l'Exposition, demanda au gouvernement de soutenir le projet de la SAP consistant à ouvrir des laboratoires ou stations dans les hôpitaux et prisons coloniales afin de permettre les études sur le terrain. L'anthropologie n'est pas une science qu'on puisse étudier en chambre, rappela Broca aux congressistes, qui votèrent la motion à l'unanimité ${ }^{1}$. Les anthropologues participèrent ainsi à l'engagement général des naturalistes de la $\mathrm{III}^{\mathrm{e}}$ République dans un inventaire et une exploitation rationnelle des ressources des territoires étrangers ${ }^{2}$.

Le fait d'établir le degré de civilisation d'une race, ou, plus généralement, les capacités mêmes d'une race à développer une civilisation, comportait des implications politiques dans le domaine colonial. En effet, le groupe des matérialistes, aux positions scientifiques homogènes, se caractérisait dans le panorama de l'anthropologie française d'après la guerre de 1870 par son militantisme. Leur activisme en faveur d'une colonisation scientifique, était la conséquence de la proximité entre leur travail scientifique et leur engagement politique aux côtés de la III ${ }^{\mathrm{e}}$ République ${ }^{3}$.

Dans le programme éditorial de la revue L'Homme (1884-1887), à laquelle Bordier participa, la géographie médicale était définie comme l'action du climat et des phénomènes atmosphériques sur le développement humain ${ }^{4}$. Elle était donc une composante importante du projet de colonialisme scientifique auquel Bordier contribuait depuis 1876, quand la SAP l'avait chargé de rédiger le questionnaire pour les médecins des hôpitaux de la Marine dans les colonies ${ }^{5}$. Selon Bordier, la colonisation française aurait été scientifique en suivant les démarches de cette discipline si utile pour formuler des règles pour l'acclimatation des migrations européennes dans les pays colonisés, essentielle pour connaître et ensuite pouvoir modifier suivant les lois de la science le milieu colonial $^{6}$. Ce sont là les lignes directrices qui guident l'œuvre publiée par Bordier en 1884, La colonisation scientifique et les colonies françaises, conçue pour réfuter la thèse de l'impossibilité d'une acclimatation européenne en Algérie, avancée par certains anthropologues dont Jean-Christian Boudin (1806-1867), médecin militaire et membre de la SAP. Ses études démographiques avaient énoncé l'impossibilité de l'acclimatation des Européens en

\footnotetext{
${ }^{1}$ R. Virchow, Création de laboratoires d'anthropologie dans les colonies.

${ }^{2} \mathrm{Cf}$. Ch. Bonneuil, Le Muséum national d'histoire naturelle ... .

${ }^{3}$ Cf. P. Desmet, La linguistique naturaliste en France (1867-1922), p. 215.

${ }^{4}$ Cf. N. Richard, La revue «L'Homme» de Gabriel de Mortillet.

${ }^{5} \mathrm{Cf}$. Comptes rendus de la V séance du congrès d'Anthropologie de Paris, Quatrième section, p. 369.

${ }^{6}$ A. Bordier, La colonisation scientifique et les colonies françaises, p. 187.
} 
Algérie. Appelé à donner une expertise à la Chambre des députés en 1847, Boudin avait contribué à faire rejeter le projet de loi sur l'implantation de colonies agricoles en Algérie ${ }^{1}$. Boudin rejetait encore dans les années 1860, dans son Traité de géographie médicale, la possibilité d'une acclimatation des Français en Algérie. À l'époque de Boudin, selon Claude Blanckaert, les anthropologues ne soutiennent pas la colonisation ${ }^{2}$. En revanche, trente ans après, pour les matérialistes, l'étude analytique des groupes raciaux aurait dû se traduire par le développement de politiques appropriées à une harmonisation des groupes qui composent la population coloniale. Ils étaient favorables à l'acclimatation' française par 'mélange des sangs', puisqu'elle pouvait servir la loi $d u$ progrès en contribuant à l'élévation des autres races vers les accomplissements de la civilisation européenne ${ }^{3}$. La race vivace et intelligente des Kabyles, dont des photographies affichées sur les murs de l'exposition anthropologique en 1878 montraient le plus beau type, était appelée à jouer un rôle important dans ce cadre ${ }^{4}$.

Une des retombées ultérieures de l'activité de Bordier à l'Exposition, fut la publication en 1881 d'une étude anthropologique sur 36 crânes d'assassins guillotinés exposés par la SAP en $1878^{5}$. Dans cette œuvre, Bordier se donnait comme programme méthodologique la comparaison du 'dossier judiciaire' avec le 'dossier anatomique' juxtaposés, pour une démonstration de la dolichocéphalie des criminels, qui sont nés avec des caractères qui étaient propres aux races préhistoriques, des caractères désormais présents seulement dans les crânes des criminels, pour une sorte d"atavisme.6. Claude Blanckaert a déjà décrit la trajectoire qui mènera, en France, à la marginalisation disciplinaire de l'anthropologie anatomique des criminels à la fin des années quatre-vingt du $\mathrm{XIX}^{\mathrm{e}}$ siècle ${ }^{7}$. Les anthropologues de la Sociétés parisienne ouvrirent ce chantier de recherche dans l'intention d'investiguer les rapports entre physique et morale, dans le but d'abolir sur un plan naturaliste, les philosophies fondées sur la liberté morale et la responsabilité métaphysique, mais ils finirent par reconnaître que leurs théories menaient à une impasse quand ils se heurtèrent à l'impossibilité de trouver un type synthétique des variables anatomiques des crânes des criminels ${ }^{8}$.

\footnotetext{
${ }^{1}$ Cf. C. Blanckaert, La crise de l'anthropométrie, p. 120.

${ }^{2}$ Cf. C. Blanckaert, La crise de l'anthropométrie, p. 114.

${ }^{3}$ A. Bordier, Rapport sur l'Ethnologie de l'Asie orientale, de l'Afrique et de l'Océanie, p. 40. Cf. aussi M. Hammond, Anthropology as a weapon of social combat in late-nineteenth-century France, p. 126.

${ }^{4}$ A. Bordier, Rapport sur l'Ethnologie de l'Asie orientale, de l'Afrique et de l'Océanie, p. 40.

${ }^{5}$ A. Bordier, Étude anthropologique sur une série de crânes d'assassins, p. 5. Quand il est nommé directeur de l'École de médecine et de pharmacie de la ville de Grenoble, après 1895, Bordier s'installa dans cette ville, sans perdre les contacts avec le groupe parisien. Il y fonda la Société dauphinoise d'ethnologie et d'anthropologie, dont il organisa le Musée.

${ }^{6}$ A. Bordier, Étude anthropologique sur une série de crânes d'assassins, p. 6, p. 12 \& p. 27.

${ }^{7}$ Cf. C. Blanckaert, De la race à l'évolution, pp. 359-393.

${ }^{8}$ C. Blanckaert, De la race à l'évolution, p. 374. Cf. aussi C. Blanckaert, De la race à l'évolution, p. 392.
} 
Les rapports entre capacités morales - dans le cas des criminels -, ou capacités intellectuelles - dans le cas des artistes dolichocéphales - et les données biologiques devaient faire l'objet d'une étude, mais les premières restaient subordonnées aux secondes. En 1878, les gravures des Lacs des Merveilles étaient utilisées par Bordier pour prouver leur manque d'intérêt dans le chantier de la recherche sur l'évolution des formes humaines.

\section{Le débat sur l'art dolichocéphale}

Par l'illustration publiée dans La Nature, Bordier expliquait donc aux lecteurs que la succession des peuples par destruction était due à la supériorité technique et sociale des brachycéphales, race d'agriculteurs et de guerriers. Ces types d'activités ne leur laissaient pas le temps, le loisir dans la définition de Bordier, pour les activités artistiques liées à l'oisiveté que pratiquaient les peuples chasseurs nomades, dont Cro-Magnon était le type ${ }^{1}$. Les traces liées à leurs productions culturelles, comme l'art, ne lui apparaissaient donc pas pertinentes dans le discours sur l'évolution humaine. Il faut bien convenir, si entaché d'utilitarisme que ce jugement puisse paraître, que cela a été un progrès, et que l'art seul ne fonde rien, telle était la conclusion de Bordier ${ }^{2}$.

Ainsi, Bordier introduisait le lecteur au centre d'un des débats les plus actuels de sa discipline, c'est-à-dire la question des capacités artistiques reconnue aux peuples primitifs, qui se prolongea après la découverte des pièces ornées par Édouard Lartet (1801-1871) et Henry Christy (1810-1865) en $1864^{3}$

L'anthropologue anglais Edward Burnett Tylor (1832-1917) avait souligné que l'art imitatif retrouvé sur les outils ornés paléolithiques ne pouvait pas être utilisé comme marqueur du degré de civilisation du peuple qui l'avait produit. Le progrès technique étant uniforme, l'on pouvait comparer les stades analogues de peuples éloignés dans le temps et dans l'espace ayant obtenu, par exemple, la maitrise des métaux; cependant, les capacités artistiques des peuples ne suivaient aucunement une trajectoire linéaire ${ }^{4}$.

Le préhistorien anglais John Lubbock (1834-1913) consacrait le chapitre Art and Ornaments de son The Origin of Civilisation (1870) aux formes d'art des sociétés plus primitives. Les ornements corporels (tatouages, modelage du crâne etc.) étaient une de leurs coutumes les plus communes. Mais si, selon Lubbock, l'art, appliqué à l'ornementation personnelle, était très répandu parmi les sauvages, l'art imitatif ne l'était pas ${ }^{5}$. Ce type d'art était, dans la définition de Lubbock, un caractère ethnologique (ethnological character) ${ }^{6}$. Certaines races en étaient dépourvues. Dans une seule région de l'Afrique du Sud, faisait

\footnotetext{
${ }^{1}$ A. Bordier, Les sciences anthropologiques à l'exposition universelle, p. 214.

${ }^{2}$ A. Bordier, Les sciences anthropologiques à l'exposition universelle, p. 359.

${ }^{3}$ É. Lartet \& H. Christy, Reliquice Aquitanica.

${ }^{4} \mathrm{Cf}$. E. B. Tylor, The condition of prehistoric races ... .

${ }^{5}$ J. Lubbock, The Origin of Civilisation, p. 46.

${ }^{6}$ J. Lubbock, The Origin of Civilisation, pp. 30-34.
} 
par exemple valoir Lubbock, les Cafres avaient une grande difficulté à comprendre la perspective mais produisaient des incisions d'animaux sur leurs outils, tandis que les Boschimans étaient capables soit de dessiner, soit de comprendre des dessins en perspective ${ }^{1}$. Finalement, comme l'art au Paléolithique avait un caractère réaliste que l'art du Néolithique ne possédait pas reproduisant des formes schématiques et géométriques -, cela prouvait pour Lubbock l'arrivée d'une race différente sur le sol européen ${ }^{2}$.

Le débat sur l'art préhistorique et sauvage ne se développa pas davantage dans les cercles savants anglais, tandis qu'en France il prenait plus d'importance. La figure même que nous analysons en est une preuve. En France, les caractères physiques des ossements retrouvés dans l'abri de Cro-Magnon ne faisaient plus de doute pour les anthropologues de la SAP. En revanche, une nouvelle problématique émergeait: comment associer l'image d'un homme anatomiquement primitif, le dolichocéphale de Cro-Magnon, à la production, par ce même homme, d'objets finement gravés comme ceux mis au jour par Lartet et Christy en 1864 dans cette même région de Dordogne?

Dans l'interprétation reprise par Broca, ces objets étaient rapportés à un art envisagé comme un produit des loisirs de la vie préhistorique. Ces hommes, primitifs mais perfectibles, surent accroître leurs connaissances, développer leur industrie et, plus encore, s'élever jusqu'à la culture des arts $^{3}$. Cette trajectoire évolutive fut interrompue au néolithique. L'art préhistorique, comme l'explique Margaret Conkey, était reconnu comme preuve d'une sensibilité esthétique, mais pas comme preuve d'une production symbolique traduisant la profondeur cognitive et active de ses auteurs ${ }^{4}$.

Bordier jugeait que les découvertes des pièces ornées préhistoriques à la Madeleine et à Laugerie-Basse par Lartet et Christy avaient engendré un débat disproportionné sur les capacités symboliques de l'homme de Cro-Magnon. Selon lui, focaliser les recherches sur ces facteurs pouvait induire en erreur, comme le démontrait le cas des Boschimans. Il considérait que le réalisme de ces représentations, c'est-à-dire la compétence artistique permettant de se faire une idée très juste et donc de reproduire la nature environnante, n'était pas suffisante pour assurer la survie d'un peuple ${ }^{5}$. C'est donc à ce moment que les copies des gravures des Boschimans exposées par la colonie du Cap attirèrent l'attention de Bordier. En effet, elles pouvaient être déterminantes dans la controverse sur les capacités artistiques des primitifs, et Bordier les utilisa pour essayer de trancher la question. Il précisa sa position face aux collègues réunis au Congrès des sciences anthropologiques d'août 1878:

Lorsqu'on compare ces dessins, qui dénotent des qualités artistiques réelles, à l'état précaire de la

${ }^{1}$ Cf. J. Lubbock, The Origin of Civilisation, pp. 35-36. Sous le nom de Cafres l'on désignait, en Angleterre, tous les peuples occupant la Colonie du Cap avant les Européens, sauf les Boschimans.

${ }^{2}$ Cf. J. Lubbock, The Origin of Civilisation, pp. 29-31.

${ }^{3}$ Cf. P. Broca, Sur les crânes et ossements des Eyzies, pp. 388-391.

${ }^{4} \mathrm{M}$. W. Conkey, Mobilizing ideologies, pp. 178-179: 'deep' cognitive and active symbolic production.

${ }^{5} \mathrm{Cf}$. A. Bordier, Les sciences anthropologiques à l'exposition universelle, p. 360. 
civilisation et de l'intelligence des Boschimans, ne se prend on pas à douter, Messieurs, des qualités que nous prêtons à nos ancêtres de la pierre taillée, sur le simple vu des dessins non supérieurs à ceux de l'Afrique australe, qu'ils nous ont laissés à la Madeleine ou à Laugerie? ${ }^{1}$

En cas de rencontre entre un peuple seulement artiste et un peuple pourvu d'avancées techniques, comme c'était le cas des colonisateurs anglais et hollandais, le premier était nécessairement voué à disparaitre: les Boschimans n'existeront bientôt plus que sur les rochers où ils se sont plu à graver leur image $^{2}$.

Cette nécessité historique serait, en tant que telle, vraie sans limite de temps et d'espace. Elle serait démontrée par l'actualité de la colonisation en Afrique du Sud au même titre que par la disparition, dans l'Europe préhistorique, de la race de Cro-Magnon auteur des gravures du Val d'Enfer et des exemples d'art mobilier exposés par la SAP. L'art, selon les matérialistes, n'était qu'un élément capricieux d'un ensemble plus vaste, plus déterminant, et il n'infirmait pas la grande loi du progrès. L'exclusion de l'art de la liste des caractères pertinents pour juger de l'évolution d'une race était une des caractéristiques des anthropologues libres penseurs auxquels Bordier appartenait ${ }^{3}$. De nos jours, les capacités symboliques font parties des éléments caractérisant les espèces humaines fossiles et donc entrant en ligne de compte dans calcul de leur destinée évolutive ${ }^{4}$. Or, les préhistoriens libres penseurs et matérialistes réunis autour de Gabriel de Mortillet, sceptiques vis-à-vis de toutes théories soupçonnées de faire le jeu de la métaphysique, contestaient la pertinence de tout élément nonmatériel dans le discours sur l'évolution humaine. Par conséquent, ils réduisaient l'importance des données culturelles telles que l'art et les sépultures ${ }^{5}$.

L'art préhistorique, au même titre que la religion, posait le problème de la complexité des manifestations culturelles des civilisations primitives. Ces éléments, en témoignant des capacités intellectuelles et morales des hommes fossiles, risquaient de brouiller la trajectoire linéaire d'une évolution qui procédait d'un degré plus simple vers un degré plus complexe. Pour cette raison, des anthropologues comme Mortillet et Abel Hovelacque (1843-1896) interprétèrent les sépultures préhistoriques comme autant de preuves que la religion était le signe d'un état barbare de la civilisation. L'athéisme, engendré dans la société occidentale par la science démasquant les superstitions religieuses, était ainsi pour eux une conquête de l'homme civilisé. L'alliance de

${ }^{1}$ A. Bordier, Rapport sur l'Ethnologie de l'Asie orientale, de l'Afrique et de l'Océanie, pp. 42-43.

${ }^{2}$ A. Bordier, Rapport sur l'Ethnologie de l'Asie orientale, de l'Afrique et de l'Océanie, p. 43.

${ }^{3}$ Cf. P. Desmet, La linguistique naturaliste en France (1867-1922).

${ }^{4}$ Pour un exemple de cette démarche dans le, désormais classique, débat sur le partage des caractères humains déterminant pour la survie entre Neandertal et Homo sapiens, cf. Ch. Stringer \& C. Gamble, In Search of the Neanderthals. Cf. aussi S. J. Gould, So near and yet so far.

${ }^{5}$ Cf. N. Richard, La revue «l'Homme» de Gabriel de Mortillet, p. 252. 
la science et de l'industrie devait permettre à l'humanité d'atteindre son plus haut degré d'évolution (cf. Figure 5) ${ }^{1}$.

Pour ce qui concerne l'art, Mortillet, dans l'entrée Art (paléontologie) du Dictionnaire des sciences anthropologiques, affichait en 1889 une opinion assez proche de celle de Bordier, même s'il considérait que l'art paléolithique était l'attribut, non d'une race, mais d'une époque tout entière ${ }^{2}$. En se référant à l'art mobilier, il relatait que l'usage pouvait détruire les représentations gravées sur les outils (manche en corne etc.), signe que les cornes ou les os frais étaient gravés avant de penser à l'emploi technique de l'objet. D'où la conclusion que les artistes magdaléniens se caractérisaient par un esprit sans prévoyance, tout comme les sauvages actuels ${ }^{3}$. La principale qualité de cet art, instinctif et donc naïf, mais tout à fait achevé pour son temps, était le réalisme, une représentation proche du vrai étant considérée comme le degré le plus bas sur l'échelle artistique. Comme le démontrait, dans son discours au congrès de l'AFAS en août 1878, le matérialiste Gaëtan Delaunay (1847-1885), le réalisme caractérise les représentations les plus primitives de l'art ${ }^{4}$. Les limites de cet art dolichocéphale, dont le manque de perspective et la composition maladroite des figures, étaient les traductions esthétiques des limitations de l'intelligence de l'homme fossile ${ }^{5}$.

Et Mortillet soulignait qu'au néolithique, l'art avait presque partout disparu. Un peuple plus évolué pouvait produire des œuvres plus grossières ou aucune; Bordier notera que la nouvelle population brachycéphale ne nous a pas laissé de traces artistiques. Cette race est connue pour avoir marqué les lieux mémorables, les dates importantes au moyen de ses constructions mégalithiques ${ }^{6}$. Par conséquent, l'art plastique et la civilisation ne marchent pas toujours parallèlement ${ }^{7}$. Pour les anthropologues matérialistes, l'art ne faisait donc pas partie des caractères humains soumis à la loi du progrès de l'humanité, telle qu'elle avait été définie par Mortillet ${ }^{8}$.

\section{Conclusions}

L'illustration publiée dans La Nature cristallise finalement le rôle qu'une Exposition universelle peut jouer dans les débats internes à la discipline anthropologique, favorisant la rencontre entre les gravures des Boschimans contemporains et celles de la population européenne disparue de Cro-Magnon et fournissant le support des arguments mis en œuvre dans un débat scientifique.

${ }^{1}$ Cf. A. Hovelacque, Notre ancêtre. Cf. aussi N. Richard, La fabrique du précurseur.

${ }^{2}$ Dictionnaire des sciences anthropologiques, p. 123 (Art).

${ }^{3}$ Dictionnaire des sciences anthropologiques, p. 123 (Art).

${ }^{4} \mathrm{G}$. Delaunay, Les dessins dans l'anthropologie.

${ }^{5}$ Cf. N. Richard, De l'art ludique à l'art magique, p. 61. Cf. aussi O. Moro-Abadía \& M. R. Gonzàlez Morales, L'analogie et la représentation de l'art primitif à la fin du XIX' siècle.

${ }^{6} \mathrm{~A}$. Bordier, Les sciences anthropologiques à l'exposition universelle, p. 214.

${ }^{7}$ A. Bordier, Les sciences anthropologiques à l'exposition universelle, p. 362.

${ }^{8}$ G. de Mortillet, Promenades préhistoriques à l'exposition universelle, p. 187. 
La figure de Bordier fonctionne sur plusieurs plans. Elle peut être comprise comme un exemple de la redéfinition des frontières de la discipline anthropologique au moment de l'émergence des études préhistoriques. Mais, en même temps, elle joue un rôle d'acte d'engagement social de la part d'un anthropologue matérialiste ${ }^{1}$. Bordier, en soulignant l'inévitable disparition des artistes dolichocéphales Boschimans aux prises avec la supériorité technique occidentale, vise à présenter comme légitime leur exclusion des races pouvant se mélanger avec les Européens dans les colonies. Il renseigne en outre les lecteurs de La Nature sur le rôle que l'anthropologie peut assurer dans la France contemporaine, celui d'une science utile à la colonisation.

C'est pour répondre à ces missions qu'Arthur Bordier publia son article dans La Nature, une revue de vulgarisation scientifique. S'adressant aux visiteurs de l'Exposition universelle venus admirer les progrès de la technique et de l'industrie occidentale, Bordier leur explique la place marginale de l'art dans l'évolution des sociétés, qu'ils peuvent rapporter à la marginalité des peuples dolichocéphales artistes contemporains. De plus, il fournit aux lecteurs et visiteurs une preuve visuelle de cette marginalité, chacun pouvant juger par soi-même de la grossièreté ou de la finesse des représentations et de la dégradation de la culture des Boschimans par rapport à celle des colons occidentaux. Environnés par les impressionnantes réalisations techniques exposées à Paris, l'inévitabilité de la disparition des artistes dolichocéphales ne fait pas de doute (cf. Figure 6). L'exposition fonctionne comme une démonstration in vivo des dynamiques raciales qu'on voudrait à l'œuvre dans l'espace colonial, par la superposition entre le déploiement des progrès techniques et l'évolution des races. L'artisanat le plus grossier est le propre de la race moins apte au progrès. L'illustration démontre, dans ce contexte, que d'autres éléments, tels que la culture, doivent rester en dehors du discours sur l'évolution humaine et propage cette démonstration au lectorat de la Nature.

\section{Bibliographie}

Bennett T., The Birth of the Museum. History, theory, politics, Routledge, London \& New York 1995.

Bordier A., Les sciences anthropologiques à l'exposition universelle in: La Nature, Sixième année, deuxième semestre, n. 261 à 287, 1878, pp. 129-131, pp. 210-214, pp. 358-362 \& pp. 408-410.

Bordier A., Rapport sur l'Ethnologie de l'Asie orientale, de l'Afrique et de l'Océanie in: Congrès international des sciences anthropologiques tenus à Paris du 16 au 21 août 1878, Imprimerie Nationale, Paris 1880, pp. 39-46.

Bordier A., Étude anthropologique sur une série de crânes d'assassins, Masson, Paris 1881.

Bordier A., Notice sur l'exposition des sciences anthropologiques in: Rapport administratif sur l'Exposition universelle de 1878 à Paris, Imprimerie Nationale, Paris, vol. 1, $3^{\mathrm{e}}$ partie, 1881, pp. 571-578.

\footnotetext{
${ }^{1}$ C. Blanckaert, De la race à l'évolution, p. 24.
} 
Bordier A., La colonisation scientifique et les colonies françaises, Reinwald, Paris 1884.

Béguet B., La science pour tous: sur la vulgarisation scientifique en France de 1850 à 1914, Bibliothèque du CNAM, Paris 1990.

Bensaude-Vincent B., Un public pour la science: l'essor de la vulgarisation un XIX ${ }^{\text {ème }}$ siècle in: Réseaux 58, 1993, pp. 47-66.

Blanckaert C., Les bases de la civilisation; lectures de "L'homme primitif» de Louis Figuier (1870) in: Bulletin de la Société préhistorique française 90, 1/1993, pp. 31-49.

Blanckaert C., Avant Adam. Les représentations analogiques de l'homme fossile dans la première moitié du XIX siècle in: L'homme préhistorique. Images et imaginaire, (éd.) A. Ducros \& J. Ducros, L'Harmattan, Paris 2000, pp. 25-54.

Blanckaert C., La crise de l'anthropométrie: des arts anthropotechniques aux dérives militantes (1860-1920) in: Les politiques de l'anthropologie. Discours et pratiques en France (1860-1940), (éd.) C. Blanckaert, L'Harmattan, Paris 2001, pp. 94-172.

Blanckaert C., La «météorologie du microcosme cérébral»: controverses raciologiques autour de Paul Broca et Pierre Gratiolet in: Les querelles du cerveau. Comment furent inventées les neurosciences, (éd.) C. Cherici \& J.-C. Dupont, Vuibert, Paris 2008, pp. 271-285.

Blanckaert C., De la race à l'évolution. Paul Broca et l'anthropologie française 1850-1900, L'Harmattan, Paris 2009.

Bleicher G., Extrait des procès-verbaux de la Société des sciences de Nancy, relatif aux communications faites par $G$. Bleicher sur le résultat de son étude scientifique de la Barbarie, Imprimerie de Berger-Levrault, Nancy 1877.

Bleichmar D., Visible Empire: scientific expeditions and visual culture in the Hispanic enlightenment in: Postcolonial studies 12, 4/2009, pp. 441-466.

Boetsch G. \& Ferrie J.-N., Le paradigme berbère: approche de la logique classificatoire des anthropologues français du XIX $X^{e}$ siècle in: Bulletins et Mémoires de la Société d'Anthropologie de Paris, Nouvelle série 3, vol. 1, fasc. 3-4, 1989, pp. 257-275.

Bonneuil Ch., Le Muséum national d'histoire naturelle et l'expansion coloniale de la Troisième République (1870-1914) in: Revue française d'histoire d'outre-mer 86, 1/1999, pp. 143-169.

Broca P., Sur les crânes et ossements des Eyzies in: Bulletin de la Société d'Anthropologie de Paris, $2^{\mathrm{e}}$ série, III, 1868, pp. 350-392.

Charpy M., Les «techniques archaïques». Produits d'un autre temps et produits artisanaux dans les expositions universelles in: Les expositions universelles en France au XIX $X^{e}$ siècle. Techniques. Publics. Patrimoines, (éd.) A.-L. Carré, M.-S. Corcy, , CNRS Éditions, Paris 2012, pp. 279-301.

Cataldi M., Inventing the Menton Man. Rivière's Discovery as Reflected in the French Media in: Centaurus 58, 3/2016, pp. 148-165.

Catalogue spécial de l'Exposition des sciences anthropologiques in: Exposition universelle internationale de 1878 à Paris, Imprimerie Nationale, Paris 1878. 
Chansigaud V., Scientific Illustrators in: A Companion to the History of Science, (éd.) Lightman Bernard, Wiley Blackwell, Oxford 2016, pp. 111125.

Charpy M., Les «techniques archaïques». Produits d'un autre temps et produits artisanaux dans les expositions universelles in: Les expositions universelles en France au XIX $X^{e}$ siècle. Techniques. Publics. Patrimoines, (éd.) A.-L. Carré, M.-S. Corcy, Ch. Demeulenaere-Douyère \& L. Hilaire-Pérez, CNRS Éditions, Paris 2012, pp. 279-301.

Collection des Guides Conty. L'Exposition en poche. Guide pratique, Exposition Universelle, Paris 1878.

Comptes rendus de la $V^{e}$ séance du congrès d'Anthropologie de Paris, Quatrième section in: Matériaux pour l'histoire primitive et naturelle de l'Homme, $2^{\mathrm{e}}$ série, vol. 9, 1878, pp. 369-371.

Conkey M. W., Mobilizing ideologies. Paleolithic 'art', gender trouble, and thinking about alternatives in: Woman in human evolution, (éd.) D. Hager Lori, Routledge, New York \& London 1997, pp. 172-207.

Conklin A., In the Museum of Man. Race, Anthropology and Empire in France 1850-1950, Cornell University Press, Ithaca 2013.

Coye N., En leur science et conscience. Vulgarisateurs et caution scientifique en préhistoire au XIX $X^{e}$ siècle in: L'homme préhistorique. Images et imaginaire, (éd.) A. Ducros \& J. Ducros, L'Harmattan, Paris 2000, pp. 206-224.

De la Blanchère, Les Diamants du Cap in: L'exposition universelle de 1878 illustrée: publication internationale autorisée par la Commission, $\mathrm{n}^{\circ} 145$, [sans éd. ni ville] 1878.

Delaunay G., Les dessins dans l'anthropologie in: Association française pour l'avancement des sciences, Congrès de Paris. Compte-rendu de la $7^{e}$ session, Paris, 1879, pp. 833-835.

Demeulenaere-Douyère Ch. (éd.), Exotiques expositions ... Les expositions universelles et les cultures extra-européennes, France, 1855-1937, Somogy Éditions d'art \& Archives nationales, Paris 2010.

Desmet P., La linguistique naturaliste en France (1867-1922). Nature, origine et évolution du langage, Peeters, Leuven \& Paris 1996.

Dias N., Séries de crânes et armée de squelettes: les collections anthropologiques en France dans la seconde moitié du XIX siècle in: Bulletins et Mémoires de la Société d'Anthropologie de Paris, nouvelle série 1, 34/1989, pp. 203-230.

Dias N., Le Musée d'Ethnographie du Trocadéro, 1878-1908. Anthropologie et muséologie en France, CNRS Éditions, Paris 1991.

Dias N., Nineteenth-Century French Collections of Skulls and the Cult of Bones in: Nuncius 27, 2012, pp. 330-347.

Dictionnaire des sciences anthropologiques, (éd.) O. Doin, Paris 1889.

Dunn E. J., The Bushman, Charles Griffin, London 1931.

Exposition des sciences anthropologiques, 1881 in: Rapport administratif sur l'Exposition universelle de 1878 à Paris $1,3^{\mathrm{e}}$ partie, Imprimerie Nationale, Paris 1881, pp. 193-196. 
Flour I., Les Moulages du Musée de sculpture comparée: Viollet-le-Duc et l'histoire naturelle de l'art in: L'artiste savant à la conquête du monde moderne, (éd.) A. Lafont, Presses Universitaires de Strasbourg, Strasbourg 2009, pp. 219-230.

Hammond M., Anthropology as a weapon of social combat in late-nineteenthcentury France in: Journal of the History of Behavioral Sciences 16, 1980, pp. 118-132.

Hochadel O., Carandell Baruzzi M. \& Florensa C., Scuffles, Scoops and Scams: The Construction of Prehistoric Knowledge in Newspapers in: Centaurus 58, 3/2016, pp. 135-147.

Hurel A., Des «Bushmen» en Europe? Vénus paléolithiques et négroïdes de Grimaldi dans la construction de la préhistoire française in: La Vénus hottentote, entre Barnum et Muséum, (éd.) C. Blanckaert, Publications scientifiques du MNHN, Paris 2013, pp. 291-363.

Gieryn Th., Boundary-Work and the Demarcation of Science from NonScience: Strains and Interest in Professional Ideologies of Scientists in: American Sociological Review 48, 6/1983, pp. 781-795.

Gould S. J., So near and yet so far in: New York Review of Books, 20 octobre 1994, pp. 24-28.

Hamy E.-Th., Précis de paléontologie humaine, J.-B. Baillière et fils, Paris 1870.

Hamy E.-Th., Les origines du Musée d'ethnographie. Histoire et documents, Ernest Leroux, Paris 1890.

Hovelacque A., Notre ancêtre. Recherches d'anatomie et ethnologie sur le précurseur de l'homme, Ernest Leroux, Paris 1877.

L'ouverture de l'exposition universelle de Paris, Chanson nouvelle patriotique chantée par les sociétés réunies Les Coeurs joyeux et L'union ouvrière de Seclin sur l'air Les moissonneurs, Imprimerie Six-Horemans, Lille 1878.

Lartet É. \& Christy H., Reliquice Aquitanica, Williams and Norgate, London $1865-1875$.

Les expositions universelles en France au XIX $X^{e}$ siècle. Techniques. Publics. Patrimoines, (éd.) A.-L. Carré \& M.-S. Corcy, Ch. DemeulenaereDouyère \& L. Hilaire-Pérez, CNRS Éditions, Paris 2012.

Lubbock J., The Origin of Civilisation, and the Primitive Condition of Man. Mental and Social condition of Savages, [2 ${ }^{\mathrm{ème}}$ éd.] Longmans, Green and Co., London 1870.

Ministère de l'Instruction publique, Exposition Universelle 1878. Catalogue du ministère de l'instruction publique, des cultes et des beaux-arts, Imprimerie de la société de publications périodiques, Paris 1878.

Moro-Abadía O. \& Gonzàlez Morales M. R., L'analogie et la représentation de l'art primitif à la fin du XIX $X^{e}$ siècle in: L'Anthropologie 109, 4/2005, pp. 703-721.

Mortillet G. de, Promenades préhistoriques à l'exposition universelle, Reinwald, Paris 1867. 
Mortillet G. de, Promenades préhistoriques à l'exposition universelle in: Matériaux pour l'histoire positive et philosophique de l'homme, vol. 3, 1868, pp. 181-368.

Moser S., The Visual Language of Archeology: A Case Study of the Neanderthals in: Antiquity 66, 1992, pp. 831-844.

Moser S., Making expert Knowledge through the Image. Connections between Antiquarian and Early Modern Scientific Illustration in: Isis 105, 2014, pp. 58-99.

Müller-Scheessel N., Fair Prehistory: archaeological exhibits at French "Expositions Universelles" in: Antiquity 75, 2001, pp. 391-401.

Noël B., Les antiquités à l'exposition universelle de Paris, en 1878 in: Les expositions universelles. Les identités au défi de la modernité, (éd.) Ch. Demeulenaere-Douyère \& L. Hilaire-Pérez, Presses Universitaires de Rennes, Rennes 2014, pp. 79-100.

Notice sur le Muséum d'ethnographie des missions scientifiques, rédigée par chacun des missionnaires scientifiques sur les objets qu'il a rapportés, Palais de l'Industrie, Paris 1878.

Picaud A., Le docteur Arthur Bordier in: Bulletin de la société dauphinoise d'ethnologie et d'anthropologie 1-2/1910, pp. 12-13.

Quiblier Ch., L'exposition préhistorique de la Galerie de l'Histoire du travail en 1867. Organisation, réception et impacts in: Les Cahiers de l'École du Louvre 5, 2014 [en ligne: http://cel.revues.org/470, consulté le 25 avril 2018].

Richard N., La revue «l'Homme» de Gabriel de Mortillet. Anthropologie et politique au début de la Troisième république in: Bulletins et Mémoires de la Société d'anthropologie de Paris, nouvelle série 1, 1989, pp. 231-255.

Richard N., De l'art ludique à l'art magique. Interprétations de l'art pariétal au XIX ${ }^{e}$ siècle in: Bulletin de la Société préhistorique française 90, 1/1993, pp. 60-68.

Richard N., La fabrique du précurseur in: Des sciences contre l'homme, vol. 1, (éd.) C. Blanckaert, Autrement, Paris 1993, pp. 64-79.

Rivière E., Gravures sur rocher du Lac des Merveilles au Val d'Enfer (Italie): Association française pour l'avancement des sciences, Congrès de Paris. Comptes rendus de la $7^{e}$ session, Au secretariat de l'Association, Paris 1879, pp. 783-793.

Rudwick M. J. S., Scenes from Deep Time, The University of Chicago Press, Chicago \& London 1992.

Schlanger N., Series in progress: antiquities of nature, numismatics and stone implements in the emergence of prehistoric archaeology in: History of Science 48, 2010, pp. 344-369.

Schlanger N., Boucher de Perthes au travail. Industrie et préhistoire au XIX siècle in: Histoire des sciences et des savoirs, vol. 2: Modernité et globalisation, (éd.) D. Pestre, K. Raj \& O. Sibum, Seuil, Paris 2015, pp. 267-283.

Séance d'ouverture, le vendredi 16 août 1878 in: Congrès international des sciences anthropologiques tenus à Paris du 16 au 21 août 1878, Imprimerie Nationale, Paris 1880, pp. 17-24. 
Sibeud E., La fin du voyage. De la pratique coloniale à la pratique ethnographique (1878-1913) in: Les politiques de l'anthropologie. Discours et pratiques en France (1860-1940), (éd.) C. Blanckaert, L'Harmattan, Paris 2001, pp. 173-198.

Simonin L., Les Missions scientifiques in: L'art et l'industrie de tous les peuples à l'Exposition Universelle de 1878, Librairie Illustrée, Paris 1878, pp. 605607.

Sommer M., Mirror, Mirror on the Wall: Neanderthal as Image and "Distortion" in Early $20^{\text {th }}$-century French Science and Press in: Social Studies of Science 36, 2/2006, pp. 207-240.

Sommer M., Human tools of the European tertiary? Artefacts, brains and minds in evolutionist reasoning, 1870-1920 in: Notes and Records of the Royal Society 65, 2011, pp. 65-82.

Stringer Ch. \& Gamble C., In Search of the Neanderthals: Solving the Puzzle of Human Origins, Thames \& Hudson, New York 1993.

Tylor E. B., The condition of prehistoric races, as inferred from observation of modern tribes in: International Congress of Prehistoric Archaeology 1868, Longmans, Green and Co., London 1869, pp. 11-25.

Thulié H., L'École d'anthropologie depuis sa fondation in: L'École d'Anthropologie de Paris, Félix Alcan, Paris 1907, pp. 1-27.

Tissandier G., Préface in: La Nature 1, 1873, pp. V-VII.

Virchow R., Création de laboratoires d'anthropologie dans les colonies in: Congrès International de Sciences Anthropologiques, tenu à Paris du 16 au 21 aout 1878, Imprimerie Nationale, Paris 1880, pp. 222-224.

Wartelle J.-C., La Société d'Anthropologie de Paris de 1859 à 1920 in: Revue d'histoire des sciences humaines 10, 2004, pp. 125-171.

Watteville J. M. B. de, Circulaire relative à la création d'un musée ethnographique in: Bulletin administratif de l'instruction publique 20, 1877, pp. 896-901.

Zerilli F., Il lato oscuro dell'etnologia: il contributo dell'antropologia naturalista al processo di istituzionalizzazione degli studi etnologici in Francia, CISU, Roma 1998. 


\section{Figures}

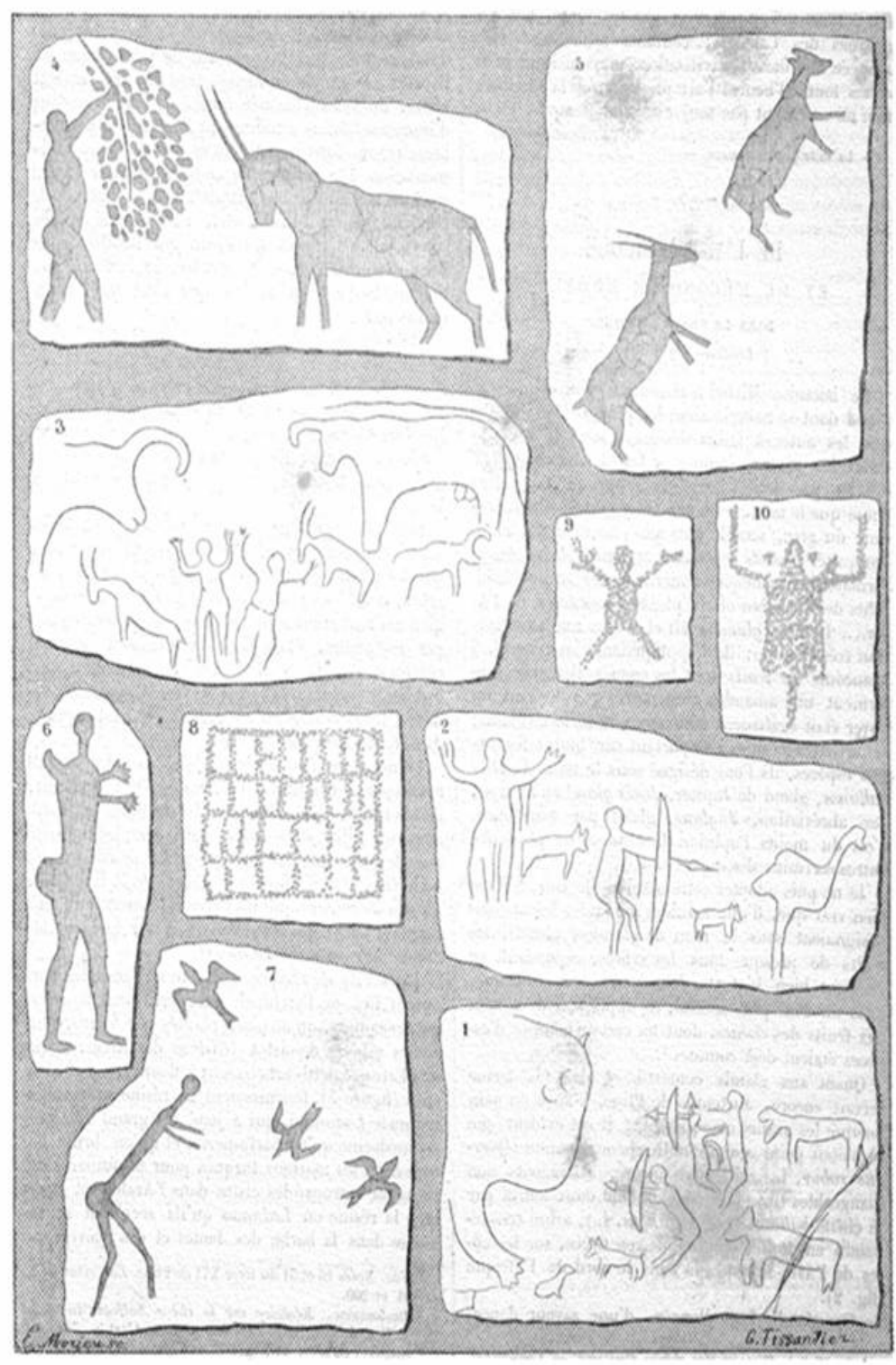

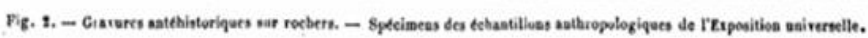

Figure 1. Gaston Tissandier, Gravures ante-historiques. Spécimens des échantillons anthropologiques de l'Exposition Universelle in: La Nature 261-287, 1878, p. 361. 


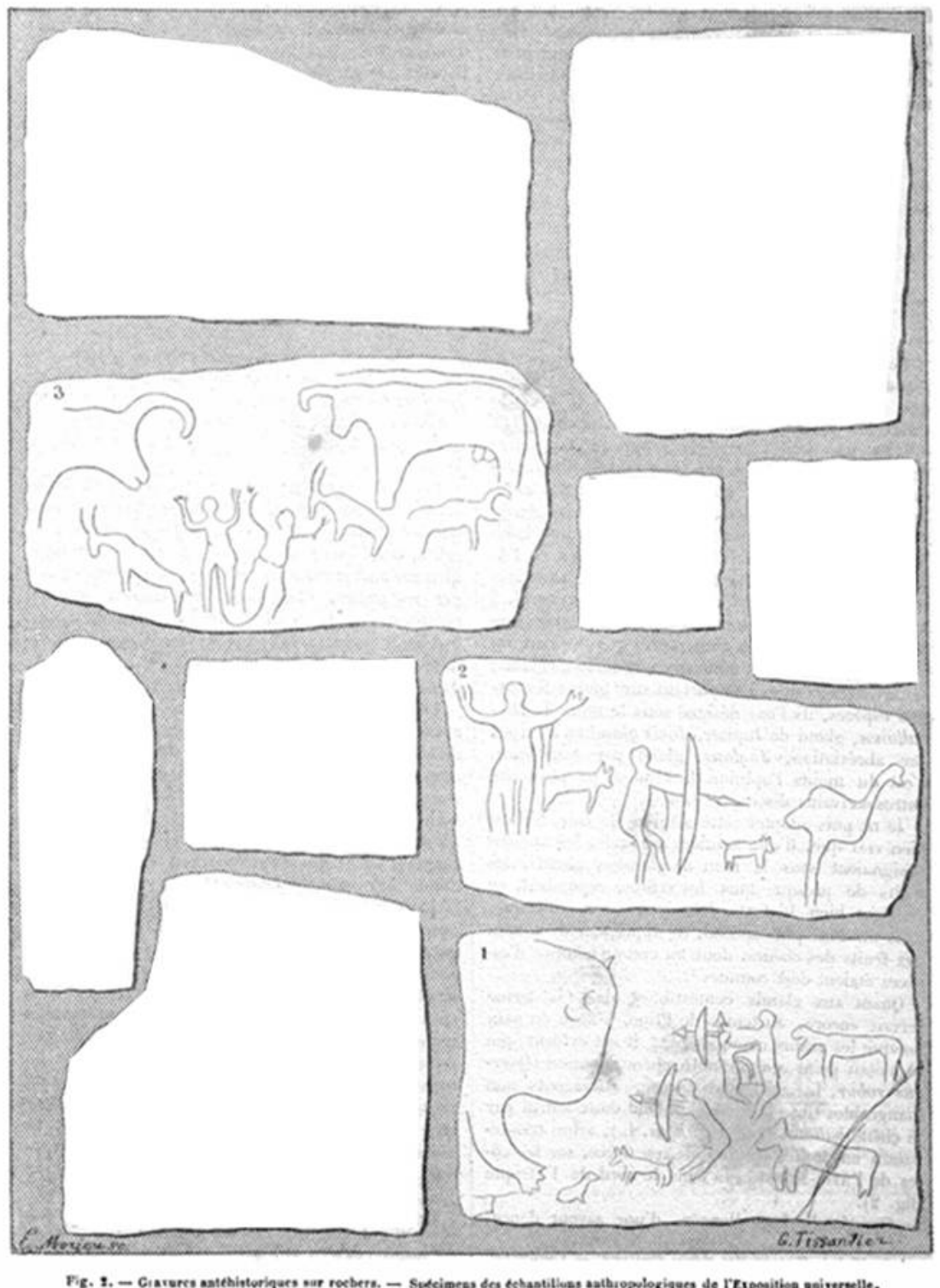

Figure 2. Les gravures de Thyout, sud-Oranais (Algérie). 


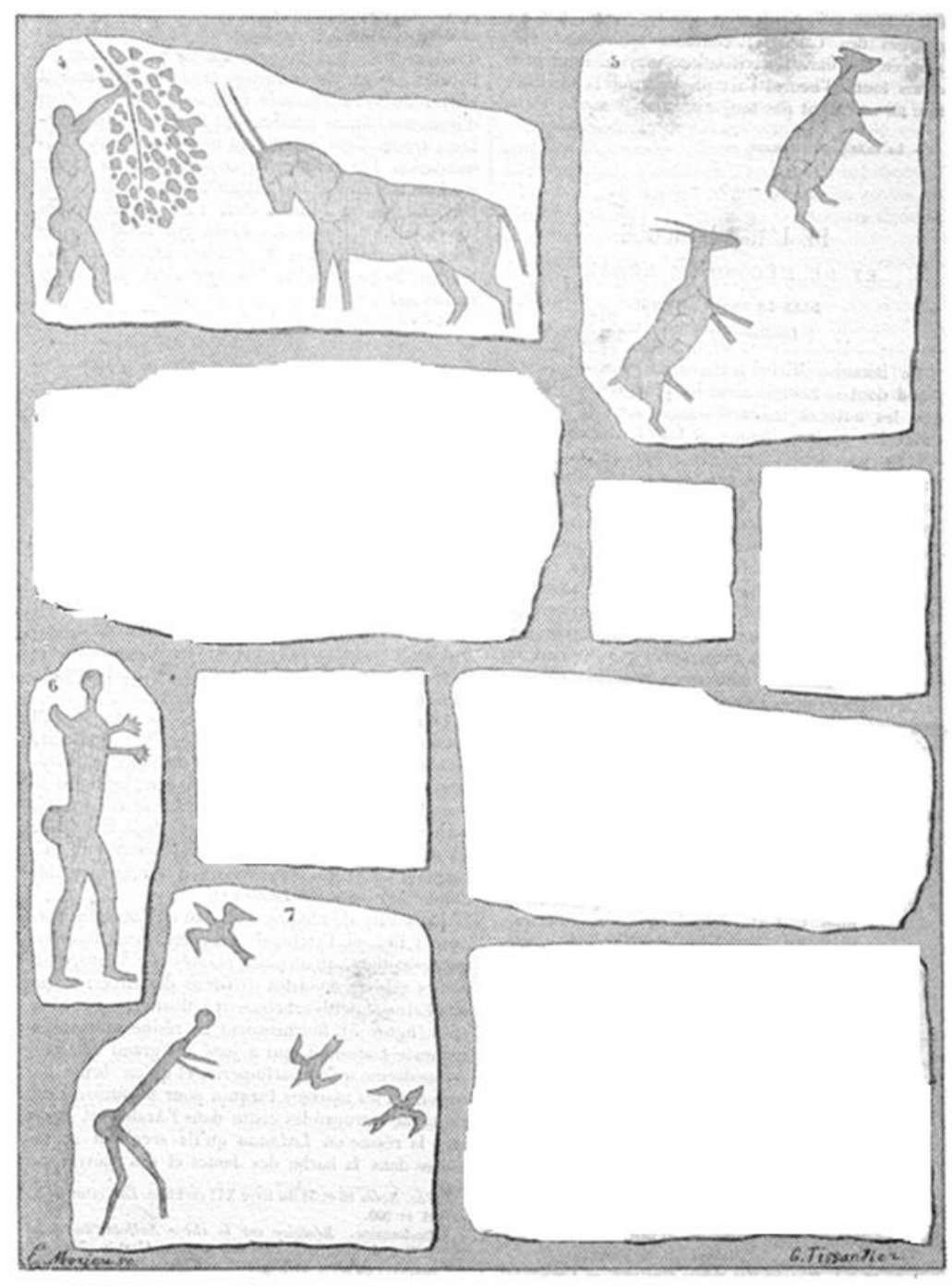

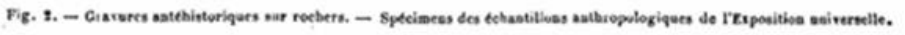

Figure 3. Gravures des Boschimans de la Colonie du Cap. 


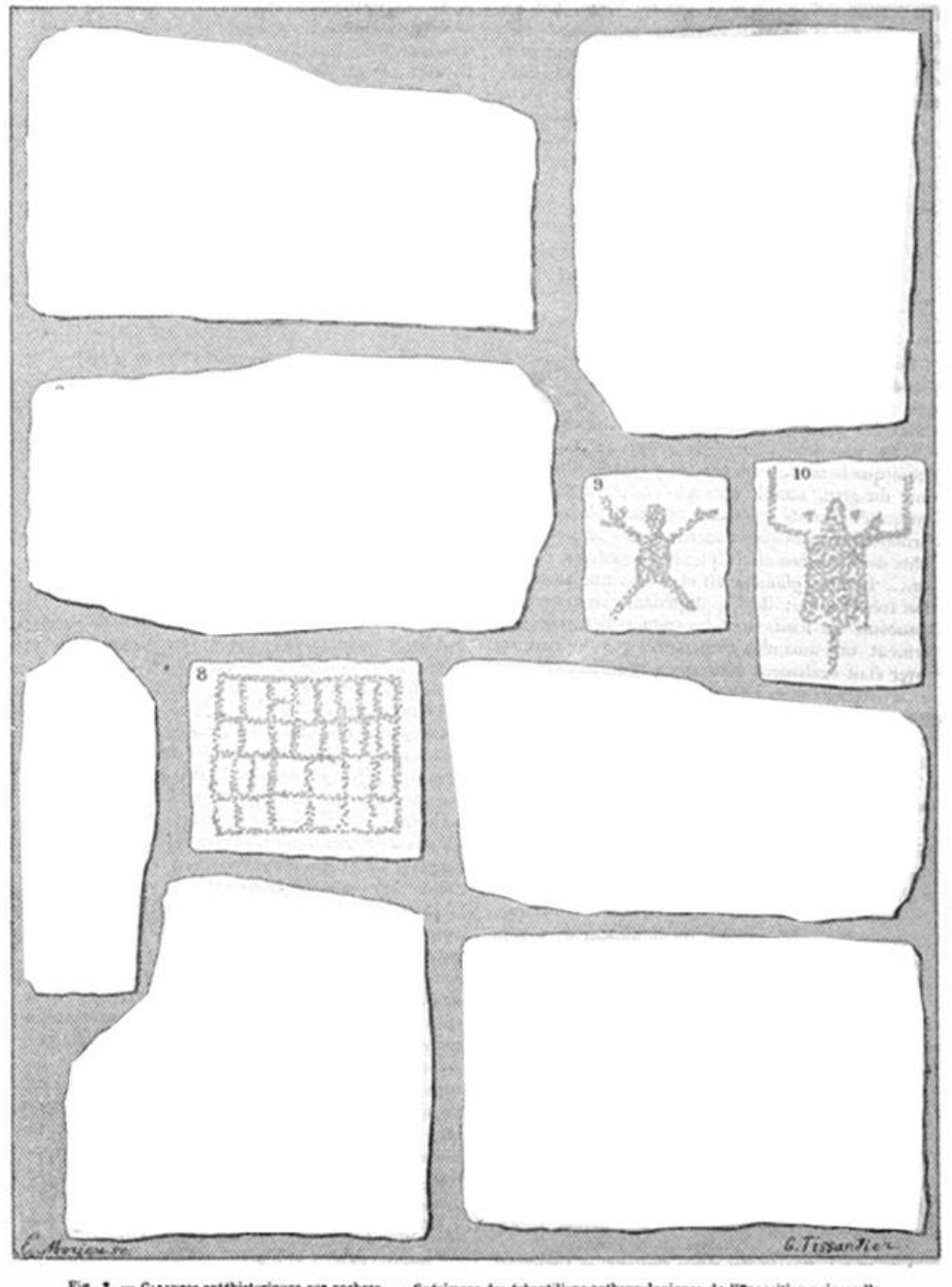

Figure 4. Les gravures des Cro-Magnon en Val d'Enfer (Italie). 


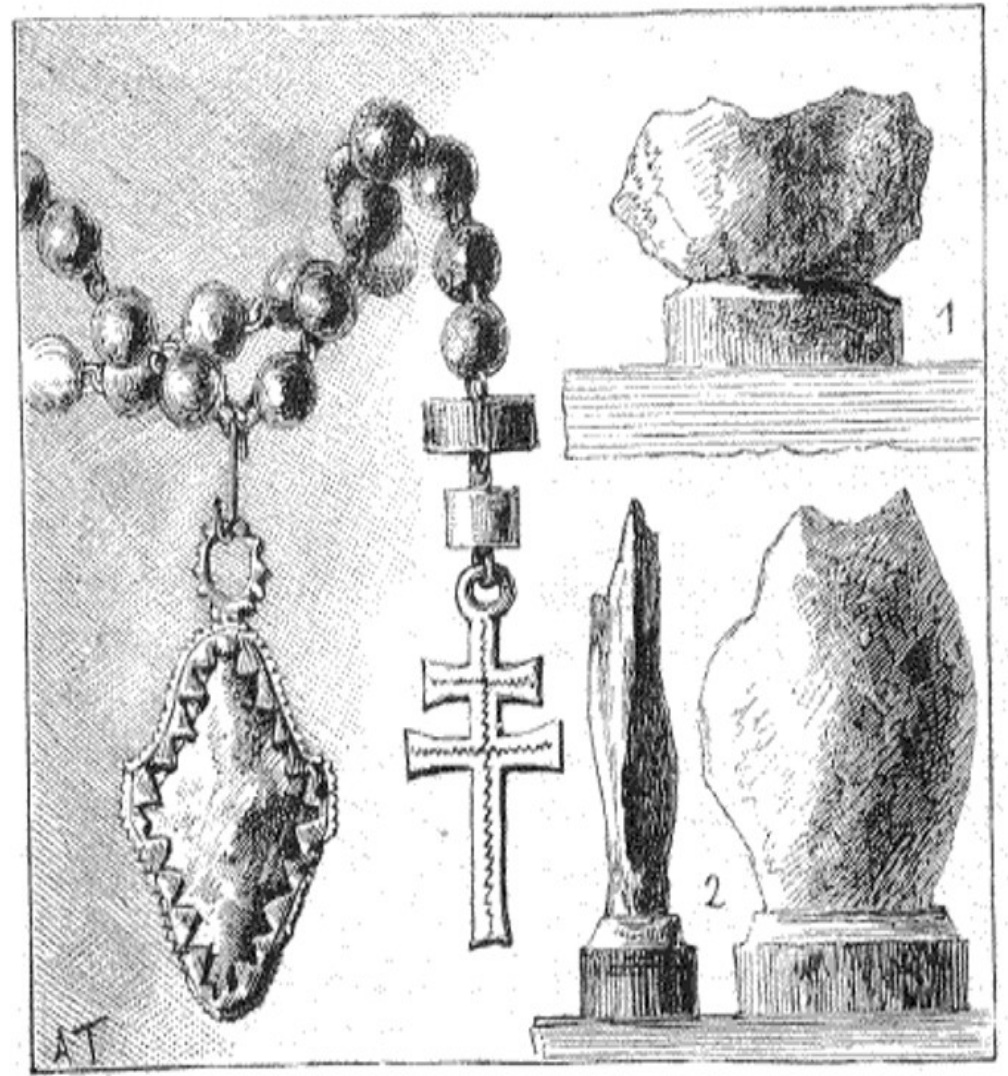

Fig. 1. $-N^{\circ} 1$ et 2, Pierres de Thenay. $-N \cdot 3$, Chapelet.

Figure 5. Pour les matérialistes, la science avait pour rôle de démasquer les superstitions religieuses. Dans ce cadre, les superstitions populaires étaient très intéressantes parce que, écrit Bordier, elles nous permettent de faire revivre la pensée des populations qui nous ont précédé. Ce chapelet, exposé et reproduit par La Nature démontre que la religion même attribuait des vertus protectrices aux armes de nos premiers ancêtres. Seule la science permet une vrai compréhension de ces objets, selon les anthropologues. La figure est dessinée par Albert Tissandier in: La Nature 261-287, 1878, p. 129; les citations sont de Bordier, p. 131. 


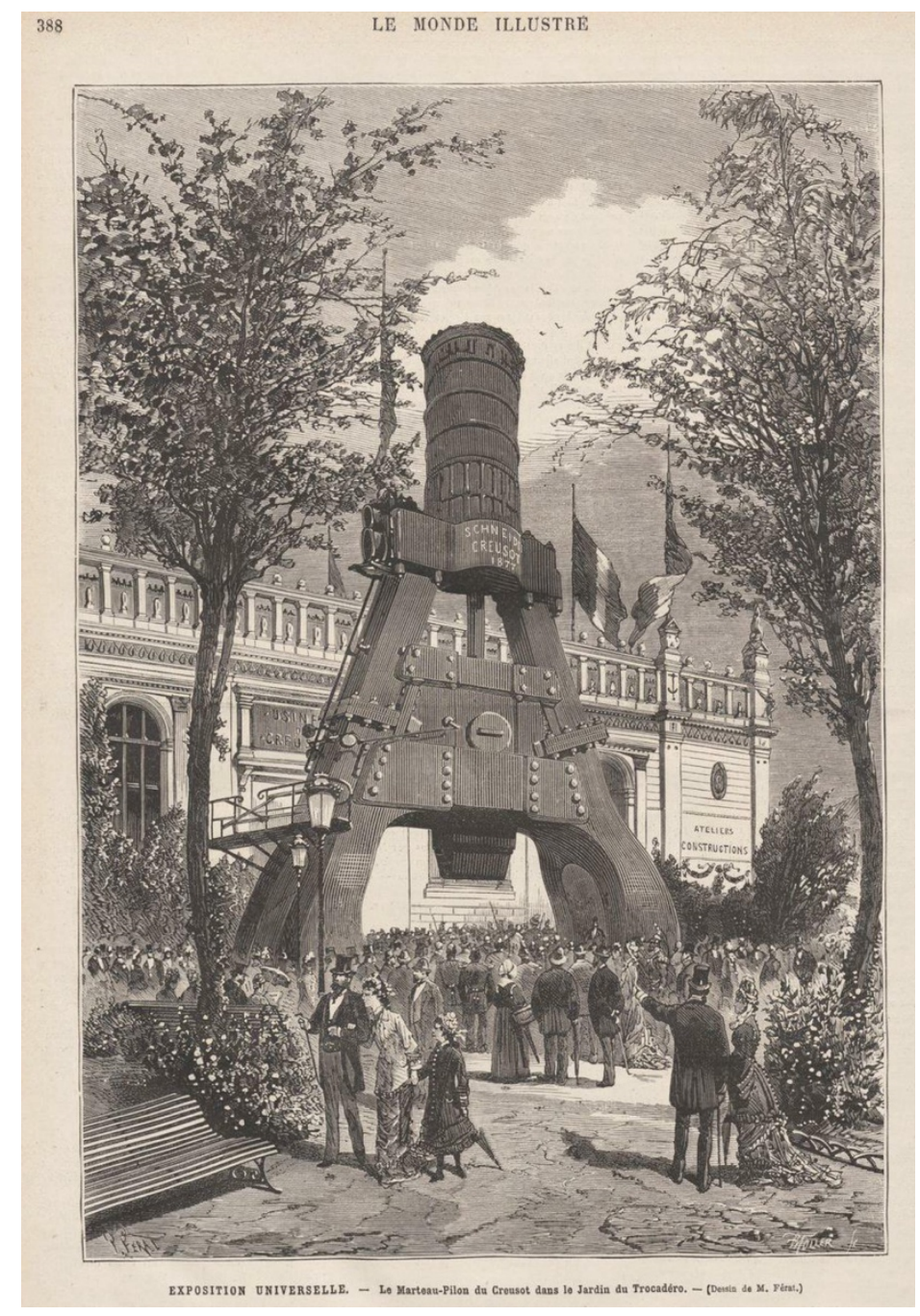

Figure 6. Le Marteau-Pilon de Creusot, exemple du progrès de la technique occidentale, exposé au Trocadéro. Dessin de M. Férat in: Le Monde Illustré du 15 juin 1878. 